\title{
Making Implementation Last: Understanding the Sustainability of an Evidence-based Treatment
}

\author{
Carrie Beth Jackson \\ West Virginia University, cbjackson@mix.wvu.edu
}

Follow this and additional works at: https://researchrepository.wvu.edu/etd

Part of the Clinical Psychology Commons

\section{Recommended Citation}

Jackson, Carrie Beth, "Making Implementation Last: Understanding the Sustainability of an Evidencebased Treatment" (2020). Graduate Theses, Dissertations, and Problem Reports. 3945.

https://researchrepository.wvu.edu/etd/3945

This Dissertation is protected by copyright and/or related rights. It has been brought to you by the The Research Repository @ WVU with permission from the rights-holder(s). You are free to use this Dissertation in any way that is permitted by the copyright and related rights legislation that applies to your use. For other uses you must obtain permission from the rights-holder(s) directly, unless additional rights are indicated by a Creative Commons license in the record and/ or on the work itself. This Dissertation has been accepted for inclusion in WVU Graduate Theses, Dissertations, and Problem Reports collection by an authorized administrator of The Research Repository @ WVU.

For more information, please contact researchrepository@mail.wvu.edu. 
Making Implementation Last: Understanding the Sustainability of an Evidence-based Treatment

Carrie B. Jackson, M.S.

Dissertation submitted to the

Eberly College of Arts and Sciences at West Virginia University

in partial fulfillment of the requirements for the degree of

Doctor of Philosophy

in Psychology

Amy D. Herschell, Ph.D., Chair

Thomas Bias, Ph.D.

Elisa Krackow, Ph.D.

Aaron Metzger, Ph.D.

Department of Psychology

\author{
Morgantown, WV
}

2018

Keywords: evidence-based treatments, implementation, training, sustainability

Copyright 2019 Carrie Beth Jackson 


\begin{abstract}
Making Implementation Last: Understanding the Sustainability of an Evidence-based Treatment Carrie Beth Jackson

Although advances have been made in facilitating the implementation of evidence-based treatments, little is known about the most effective way to sustain their use over long periods of time. Prior systematic reviews and research have suggested that organizational characteristics and training methods may be strategies that support sustainability, yet this has remained relatively unstudied in the field of behavioral health. The current study examined the sustainability of Parent-Child Interaction Therapy following a statewide implementation trial testing three training methods. Participants included 100 clinicians and 50 administrators from 50 organizations across Pennsylvania. Multi-level path analysis was utilized to examine the role of organizational barriers and training on sustainability. Clinicians and administrators reported high levels of sustainability at 24-months (12-months post-training) in the current study. Several organizational variables, including training exposure and utilization and resources at baseline and following training were associated with greater sustainability. The cascading model training condition was also related to being more likely to sustain the use of Parent-Child Interaction Therapy. Possible mechanism of change, through increased organizational resources, were also identified. Implications for training and the broader field of implementation science are discussed.
\end{abstract}




\section{Table of Contents}

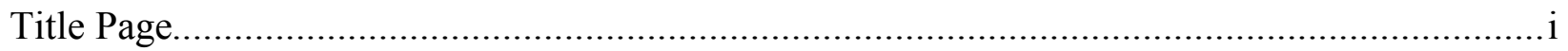

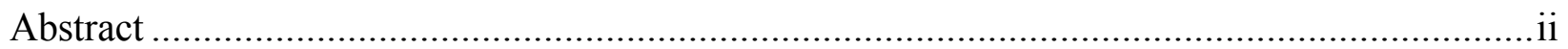

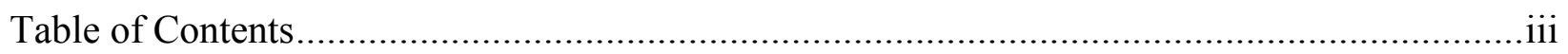

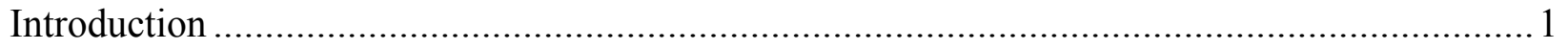

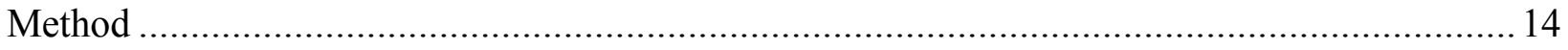

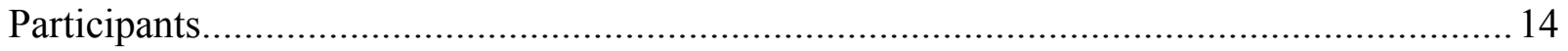

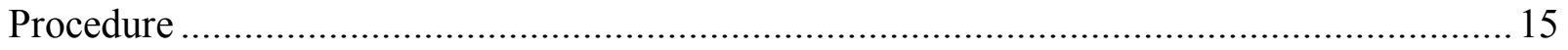

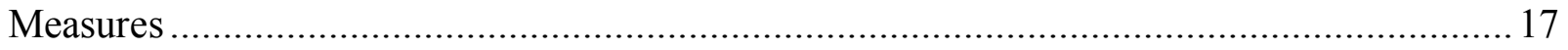

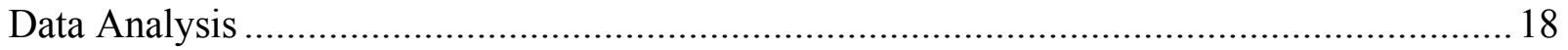

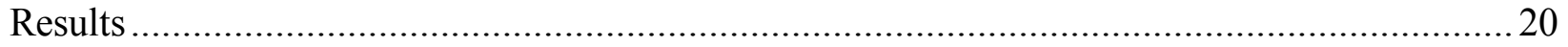

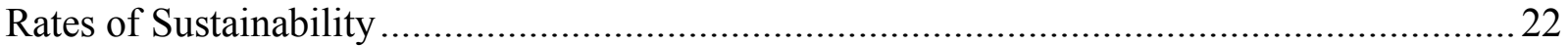

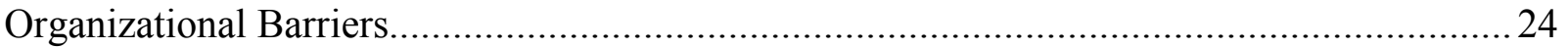

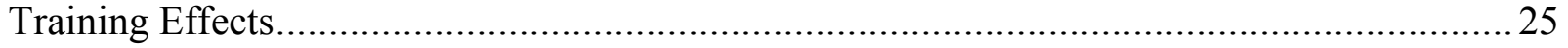

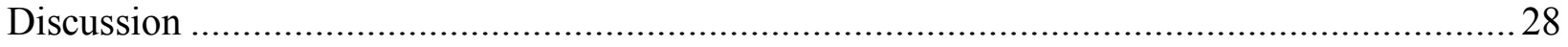

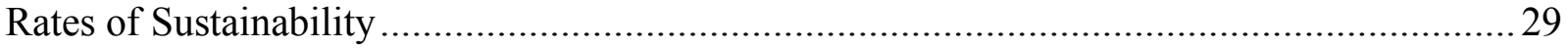

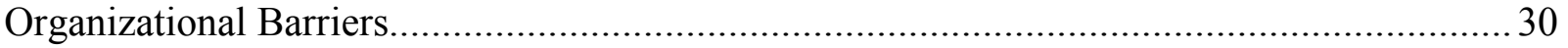

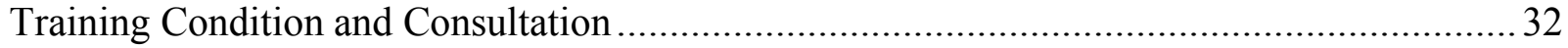

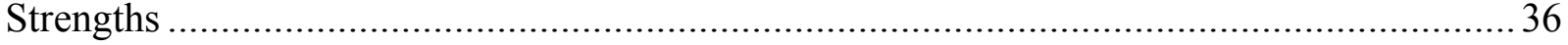

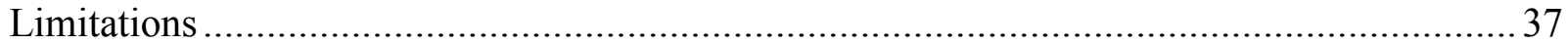

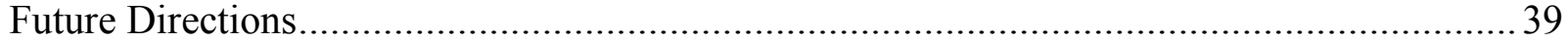

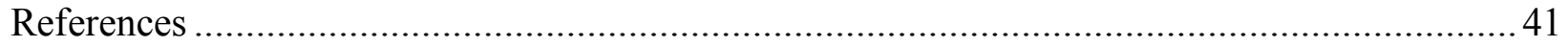

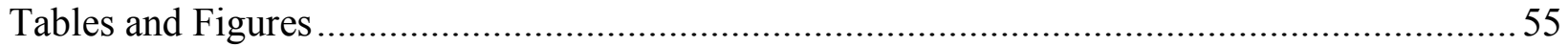


Making Implementation Last: Understanding the Sustainability of an Evidence-based Treatment Evidence-based Treatments

Mental health disorders are the most prominent health conditions for children and adolescents; however, it has been estimated that approximately $70 \%$ of these individuals never receive the mental health services they need (Kazdin, 2017; Perou et al., 2013). Of those that do receive services, a majority do not receive evidence-based treatments (EBTs) (Kazdin, 2018). EBTs refer to treatments that have been tested extensively through rigorous research and are preferred treatment approaches by organizations such as the American Psychological Association (APA Presidential Task Force on Evidence-Based Practice, 2006). Researchers have continued to make significant progress in the development of EBTs for a variety of mental health disorders within the past decade (Dorsey et al., 2017; Evans, Owens, \& Bunford, 2014; Higa-McMillan et al., 2016; Kaminski \& Claussen, 2017; Weersing et al., 2017).

\section{Research-practice Gap}

Despite these advances, EBTs are not commonly available in community settings where many families receive their mental health services. A commonly cited barrier contributing to this gap is that mental health providers may not have adequate training in the use of EBTs (Kazdin, 2018). Similarly, clinicians may be more likely to rely on anecdotal evidence and clinical intuition rather than research when making treatment decisions in clinical practice (Steele, Elkin, \& Roberts, 2008; Stewart \& Chambless, 2007). Additionally, clients seen in community settings may differ from those in the university settings in which EBTs are typically developed, as they often have more comorbidities and greater problem severity (Southam-Gerow, Chorpita, Miller, \& Gleacher, 2008; Southam-Gerow, Weisz, \& Kendall, 2003). These differences between 
research and practice settings have led to a unique challenge of increasing access to EBTs in community settings.

\section{Implementation Science}

The field of implementation science focuses on identifying methods that facilitate the transfer of EBTs into community settings (Proctor et al., 2009). Implementation is defined as the "use of strategies to introduce or change evidence-based health interventions within specific settings." A growing body of research indicates that in addition to creating and testing EBTs, research must also understand and test active implementation strategies to increase the uptake of EBTs (U.S. Department of Health and Human Services, 2006). A variety of implementation strategies have been developed to improve implementation, including planning, educational, financial, restructuring, and quality management strategies; however, the effectiveness of these strategies has rarely been researched (Powell et al., 2013; Powell et al., 2015).

\section{Sustainability}

After the goal of initially implementing a treatment within a given setting is achieved, the focus then shifts to sustainment. Sustainability is defined as the "extent to which a newly implemented treatment is maintained or institutionalized within a service setting's ongoing, stable operations" (Proctor et al., 2011, p. 70). Sustainability is often conceptualized as a multifaceted concept, measured by whether an organization is continuing to provide a treatment, or the number of clients receiving a treatment from a clinician within an organization (Stirman et al., 2012). Although there is variability in the time-frame utilized to define sustainability, a treatment still in place two years after the initial implementation phase is typically considered to be sustained (Proctor et al., 2011; Stirman et al., 2012). Sustainability is an important concept to study within treatment outcome research, as it is essential to achieving public health goals and 
necessary for a treatment to be in place for an extended amount of time to reach a broad population (Schell et al., 2013). Despite the importance of sustainability, it has remained relatively unstudied in the field of implementation science.

Rates of sustainability. It is necessary to understand the extent to which initial implementation efforts are sustained. While relatively few programs are able to achieve implementation of an innovation, an even greater number of programs have difficulty sustaining their use (Stirman et al., 2012). A recent review of sustainability studies found that only five of twenty programs $(25 \%)$ achieved full sustainability with all initial program or intervention components still being utilized (Stirman et al., 2012). Across empirical studies, rates of sustainability range from 20 to $80 \%$, with considerable variability in the definition and measurement of sustainability (Bond et al., 2014; Cooper, Bumbarger, \& Moore, 2015; Kolko, Iselin, \& Gully, 2011; Lamontagne, Swaine, Lavoie, \& Careau, 2011; Loman, Rodriguez, \& Horner, 2010; Scheirer, 2005; Swain, Whitley, McHugo, \& Drake, 2010). Although this rate is alarming, a greater number of programs are able to partially sustain an intervention, maintaining some of the program's original components. A systematic review has found that up to $60 \%$ of programs are sustained at a reduced level (Scheirer, 2005). Similarly, qualitative research supports that newly implemented programs are more sustainable if the clinicians are able to make modifications to the treatment protocol (Palinkas et al., 2013). It has been suggested that modifications make EBTs more acceptable and sustainable for clinicians, yet it is unclear if partial sustainability is sufficient to produce positive client outcomes (Stirman et al., 2013). This is significant, given that organizations expending tremendous costs to initially implement an intervention may not reap the long-term benefits due to failed sustainability (Scheirer, 2005). 
Rates of sustainability also generally change over time. There may be an initial uptake in the amount of services provided during the first year of a new program, but the volume of clients seen typically flattens out over time (Brookman-Frazee et al., 2016). Previous research has also found that fewer programs are able to sustain interventions, the longer the time-period since funding has ended (Bond et al., 2014). Evidence also suggests that rates of sustainability differ by EBT. For example, Brookman-Frazee et al., (2016) examined rates of sustainability for six EBTs over 19 fiscal quarters. While some treatments showed positive slopes in clients seen over time, other practices, including Trauma-Focused Cognitive Behavioral Therapy, demonstrated an average negative slope over time (Brookman-Frazee et al., 2016). Therefore, it is important to understand rates of sustainability at different time-periods and across interventions.

Dynamic sustainability framework. Based upon several reviews, there is a growing amount of research indicating that programs are unable to sustain the use of an intervention following initial implementation. This has led researchers to explore factors that facilitate or hinder the sustainability of an intervention. The dynamic sustainability framework suggests that intervention characteristics, the practice setting, and ecological system, as well as changes to these factors over time, influence use of an intervention (Chambers, Glasgow, \& Stange, 2013). The intervention domain refers to treatment components as well as the way in which the intervention is delivered (i.e., face-to-face, web-based) that may impact the number of clients served. Practice setting includes aspects such as organizational culture, climate, training, availability of resources, and staffing. The ecological system includes policies, market forces, and population characteristics that may affect sustainability (Chambers et al., 2013). Importantly, this framework has not been tested so it is necessary to consider it within the context of previous research. 
Prior systematic reviews have concluded that the practice setting has an impact on sustainability (Scheirer, 2005, Stirman et al., 2012). However, as part of a review on the influences on sustainability, Stirman et al. (2012) found that contextual variables such as climate, culture, leadership, and other organizational characteristics have received the least amount of attention in the literature. Additionally, focus groups, that included quality improvement and healthcare experts, have highlighted the importance of organizational context on sustainability (Doyle et al., 2013). Healthcare experts have suggested that organizational variables (e.g., financing) can be identified prior to implementation efforts, and organizations can then make efforts to plan for sustainability (Doyle et al., 2013). Given the need to understand specific organizational variables that influence sustainability and their ability to be addressed prior to implementation, the proposed study focuses on practice setting predictors.

Practice setting predictors. Several aspects of organizations have been identified as predictors and barriers to sustainability. Organizational structure has been linked to sustainability, with smaller organizations (Abraham, Knudsen, \& Roman, 2011) and community mental health settings (Brookman-Frazee et al., 2016) having lower sustainability in comparison to larger organizations and office settings.

Organizational support in various forms has also been identified as a key predictor of sustainability. Several studies have found that greater administrative support facilitates sustainability (Loman et al., 2010; Tibbits, Bumbarger, Kyler, \& Perkins, 2010). Other studies have identified having available resources, specifically adequate funding (Loman et al., 2010; McIntosh, Kim, Mercer, Strickland-Cohen, \& Horner, 2014) and training opportunities (LaPelle, Zapka, \& Ockene, 2006; Loman et al., 2010) as facilitators of sustainability. Inadequate funding is consistently cited as a barrier to sustainability; however, organizations that initially develop a 
fiscal plan outlining program needs are more likely to continue using an intervention (Cooper et al., 2015).

Aspects of an organization's culture and climate have been examined in relation to sustainability. A review by Scheirer (2005) found that a majority of organizations were able to sustain an intervention when it aligned with the existing goals and structure of the organization. Similarly, having a positive implementation climate, where an organization supports clinicians' use of an intervention, has been associated with higher rates of sustainability (Lyon, Frazier, Mehta, Atkins, \& Weisbach, 2011). This aligns with other research on how organizational factors affect sustainability. Together, these results suggest that when clinicians are part of an organization in which their use of an intervention is rewarded and supported, they are more likely to continue to use the intervention past the initial implementation stage.

Staffing attributes within an organization may also affect the sustainability of an intervention. As noted previously, clinicians may make modifications to a treatment which increases its acceptability to clinicians and subsequent sustainability (Palinkas et al., 2013; Stirman et al., 2013). Clinician attitudes may also affect sustainability, with clinicians who have more positive attitudes towards a new treatment and implementation process being more likely to sustain the use of an EBT (Lyon et al., 2011; Stirman et al., 2012). Clinician turnover may also affect rates of sustainability, with higher clinician turnover being related to lower sustainability (Bond et al., 2014; Lyon et al., 2011). Given that organizations invest training resources into clinicians for implementing an EBT, the organization may be unable to sustain the EBT if its trained clinicians leave.

Overall, previous research has attempted to disentangle organizational aspects that may influence sustainability. While the research is in the beginning stage, it is likely that aspects of 
the organization's structure, adequate funding and resources, opportunities for training, positive organizational climate, as well as attributes of the staff (i.e., lower turnover, more positive attitudes) may impact sustainability. Given this literature, the current study focuses on these variables and their influence on sustainability.

\section{Training}

Training has been identified as one implementation strategy that can enhance implementation outcomes, including sustainability. Although state mental health systems have mandated the use of EBTs by clinicians, many clinicians have not been adequately trained in their use (Rodríguez, Southam-Gerow, O’Connor, \& Allin, 2014). When clinicians are inadequately trained, they are less likely to implement an EBT with fidelity; however, there is little empirical evidence on the most effective way to train clinicians (DiMeo, Moore, $\&$ Lichtenstein, 2012; Jensen-Doss, Hawley, Lopez, \& Osterberg, 2009). Despite these limitations, stakeholders have indicated that training is not only one of the most significant barriers, but is also one of the most modifiable factors affecting the implementation of EBTs (Aarons, Wells, Zagursky, Fettes, \& Palinkas, 2009). Therefore, training clinicians in the use of EBTs represents a key strategy that should be targeted to improve sustainability. Although progress has been made in understanding how training may facilitate implementation, little is known about how it influences sustainability.

Training models. Given the need to effectively train clinicians in EBTs, research has been conducted to examine the effectiveness of various training models. Within the field of behavioral health, brief workshops and reading treatment manuals are commonly utilized methods; however, they have been shown to be largely ineffective at improving clinician knowledge and skill (Herschell, Kolko, Baumann, \& Davis, 2010). Research suggests that brief 
training methods do not allow clinicians enough time to fully learn all treatment components or incorporate the EBT into their current caseload (Beidas, Edmunds, Marcus, \& Kendall, 2012). Although they are limited in their effectiveness, brief training methods continue to be utilized with the behavioral health workforce.

Learning collaborative. Several other models have been utilized to train clinicians, with the Learning Collaborative (LC), Cascading Model (CM), and Distance Education (DE) being the most frequently used methods according to one systematic review (Herschell et al., 2010). The LC was developed after the Institute for Healthcare Improvement's Breakthrough Series Collaborative Model (American Diabetes Association, 2004). It has been used extensively by the National Child Traumatic Stress Network and focuses on training multiple levels within an organization, including clinicians, supervisors, and senior leaders (Markiewicz, Ebert, Ling, Amaya-Jackson, \& Kisiel, 2006). LC models may be effective in improving clinicians' networks of advice, and serve as a means of informal support throughout implementation (Bunger et al., 2016). Compared to other training methods, the LC is costly at approximately $\$ 19,000$ per clinician (Dopp, Hanson, Saunders, Dismuke, \& Moreland, 2017). A cost-effectiveness analysis revealed that the LC model may be less cost-effective than other training approaches in improving clinician skill; however, the subsequent changes in client outcomes were highly costeffective (Dopp et al., 2017). Another study found that the LC model was ineffective at increasing provider use of preventive services (Solberg et al., 2000). Due to the limited research on the LC model, additional research is necessary to understand its impact on clinician and client outcomes.

Cascading model. The CM is a hierarchical training method where one clinician is initially trained, and is subsequently able to train other clinicians within his/her agency 
(Herschell et al., 2015). This type of approach may also be appealing to clinicians who can be trained within their organization and reduce some of the costs incurred by typical trainings (Zandberg \& Wilson, 2013). Few empirical studies have examined the efficacy of the CM (Herschell et al., 2010; Martino et al., 2010). One of the studies found that although the initially trained clinicians demonstrated improvements in behavior, supervisees trained by these clinicians had significant variability in their competency (Shore, Iwata, Vollmer, Lerman, \& Zarcone, 1995). Another study found that compared to a self-study training condition, the CM was comparable to an expert-led workshop in teaching clinicians the principles of motivational interviewing (Martino et al., 2011). A similar study found that although there were initial improvements in clinician fidelity and skill following participation in a CM training, these improvements were not maintained at a 12-week follow-up (Martino et al., 2010). While there are notable advantages to using $\mathrm{CM}$ to train clinicians, there has been little empirical evidence to support its use.

Distance education. DE training models involve clinicians independently studying training materials, often through an online platform or reviewing training videos. Similar to CM, DE methods have the potential to be cost-effective and reach a large number of clinicians (McMillen, Hawley, \& Proctor, 2016). Clinicians have also expressed satisfaction with DE methods, as they can be completed at the clinician's pace and are an alternative to more expensive training options (Powell, McMillen, Hawley, \& Proctor, 2013). Several systematic reviews have been conducted on the effectiveness of DE methods for behavioral health clinicians (Calder, Ainscough, Kimergard, Witton, \& Dyer, 2017; Jackson, Quetsch, Brabson, \& Herschell, 2018). Together, these reviews concluded that DE methods may be effective in facilitating clinician knowledge and skill under certain circumstances, but the included studies were limited 
by numerous methodological concerns making it difficult to draw conclusions (Calder et al., 2017; Jackson et al., 2018). While there has been an increased interest in the use of DE methods, additional research is needed to understand their effectiveness.

Multi-component training models. While the aforementioned training models are typical methods in behavioral health, it is becoming more common to use multi-component trainings. Multi-component training models include several training components (i.e., treatment manuals, workshops, expert consultation) and have been shown to be more effective than briefer training methods (Herschell et al., 2010; Kolko et al., 2012; Schoenwald, Sheidow, \& Letourneau, 2004). Multi-component trainings have demonstrated efficacy across several EBTs, with a recent review finding that nineteen of twenty-one studies showed a significant improvement in at least one outcomes (e.g., increased skill; Herschell et al., 2010). This has led researchers to explore the specific components that are associated with effectiveness. A key finding of studies on multicomponent training models is that they often incorporate some type of ongoing support.

Ongoing support. Ongoing support is a training strategy that involves a continuous and consistent training in various forms (Nadeem, Gleacher, \& Beidas, 2013). Several types of ongoing support exist, including supervision, feedback, and consultation. The current study uses consultation as an ongoing support strategy. Ongoing support addresses a shortcoming of brief trainings by providing ongoing training, skill-building, and direct case application through contact with an expert, supervisor, or peer (Nadeem et al., 2013). Few studies have examined the outcomes associated with participating in consultation. In a randomized controlled trial of three training conditions, training condition did not predict post-training clinician knowledge or skill; however, clinicians that participated in more consultation calls had greater improvements in skill and fidelity (Beidas et al., 2012). Another study found similar results with clinicians participating 
in more calls and having a high caseload had the most improvements in skill in an EBT (Jackson, Herschell, Schaffner, Turiano, \& McNeil, 2017). Consultation has also been linked to positive client outcomes, with clinicians participating in training and consultation for Multisystemic Therapy being more adherent to the treatment protocol, resulting in significantly decreased client problem behaviors (Schoenwald et al., 2004). With the limited research available on consultation, it appears to be a promising ongoing support strategy that may be used in conjunction with traditional training approaches.

\section{Limitations of Previous Research}

It has been acknowledged that sustainability is an important topic of research, yet most of the research-to-date has been largely theoretical in nature. Additionally, most of the research has been conducted in school settings (Stirman et al., 2015). This is problematic given that implementation science stresses the role that setting has on influencing implementation outcomes (Beidas \& Kendall, 2010). Community-based settings may differ extensively from school settings, based on characteristics of its implementers, differences in population, and varying characteristics of the setting in which the intervention is being implemented. Therefore, it is necessary to take into account the role of contextual factors to understand the sustainability of EBTs in community settings. Of the studies that have been conducted on sustainability, there are significant limitations in their design. As previously mentioned, most studies of sustainability have used a single question to measure sustainability. This is associated with a host of measurement issues with one of the primary issues being that it is unclear if a single question is capable to adequately capturing sustainability as a construct (Martinez, Lewis, \& Weiner, 2014). Finally, with regards to training, there is little empirical evidence to support training as an implementation strategy to improve sustainability, and the mechanisms through which training 
works. Together, these limitations make it clear that there is little that is known about the sustainability of EBTs in community mental health agencies.

\section{Current Study}

The current study sought to address these limitations and contribute to a growing body of literature on the sustainability of EBTs in community settings. The current study examined sustainability at 24-months (12-months post-training) by utilizing data from a statewide implementation trial of Parent-Child Interaction Therapy (PCIT).

Aim one. The first aim of the study was to describe the extent to which administrators and clinicians sustained the use of PCIT at 24-months (12-months post-training). Sustainability was measured according to previous literature; variables included the number of clients served, clinician use of a protocol, and administrator report of program continuation (Proctor et al., 2011).

It was hypothesized that approximately $60 \%$ of clinicians would still be utilizing PCIT to some extent, similar to previous reviews (Scheirer, 2005; Stirman et al., 2012). Similarly, it was hypothesized that only $20 \%$ of clinicians would report maintaining all aspects of PCIT in their clinical practice, as previous reviews found relatively low levels of full sustainability (Stirman et al., 2012).

Aim two. The second aim of the proposed study was to identify baseline organizational variables that facilitated or hindered sustainability at 24-months. Specifically, were greater baseline organizational resources, training exposure and utilization, and organizational climate associated with more sustainability at 24-months?

Previous research has suggested organizations that have more resources are more likely to sustain an intervention after funding for a project has ended (Loman et al., 2010; Tibbits et al., 
2010). These studies indicate having more resources prior to beginning a program allows for organizations to plan for sustainability in the initial implementation stages (Tibbits et al., 2010). Similarly, the impact of training has been examined, such that organizations who provide opportunities for their clinicians to receive training are more likely to sustain an intervention (Loman et al., 2010). Organizational climate has a strong conceptual background (Schneider, Ehrhart, \& Macey), and has been shown to influence a variety of implementation outcomes (Ditty et al., 2015; Beidas et al., 2014), including sustainability (Scheirer, 2005; Lyon et al., 2011). Based upon this previous research, it was hypothesized that greater resources and training exposure, along with more positive organizational climate would be related to greater clinician sustainability. Additionally, it has been acknowledged that clinician attitudes towards an intervention impact the extent to which a clinician uses an intervention (Stirman et al., 2015), therefore, clinician satisfaction with PCIT was included as a covariate in all analyses.

Aim three. The third aim tested the indirect effect of training design and consultation call attendance on sustainability through post-training resources, training exposure and utilization, and organizational climate. It was expected that participation in the Learning Collaborative and Cascading Model training designs and attending more consultation calls would be associated with a clinician having more positive perceptions of their organization's resources, training exposure and utilization, and organizational climate at post-training (12-months), given that these two designs involve training multiple members within an organization. On the other hand, clinicians in the Distance Education condition may not feel as supported by their organization, given their supervisors and administrators did not participate in a training. When a clinician perceives they are more supported by their organization through more resources, greater training exposure and utilization, and more positive organizational climate, they may be more likely to 
sustain the use of PCIT. While it was expected that training design and consultation call attendance would have a positive impact on all three of these hypothesized mediators, it was expected that they would independently affect sustainability and therefore, were studied as separate variables. Clinicians in organizations with greater resources, more positive organizational climate, and more training experience, would be more likely to provide PCIT to a greater number of clients and continue using all parts of the PCIT protocol, which are key indicators of sustainability (Proctor et al., 2011; Stirman et al., 2013).

Training has been suggested as an implementation strategy to improve implementation and sustainability (Powell et al., 2013). Similarly, a lack of training and resources has been identified as a barrier to sustainability (Loman et al., 2010). Despite the knowledge that training may impact sustainability, little is known about the types of training design and ongoing support that lead to sustainability. While some training models may primarily influence implementation by changing clinician knowledge, other training models (i.e., Learning Collaborative), may lead to organizational change by targeting multiple levels within an organization. When clinicians and their organization participate in a training, they may feel more supported by their organization, and be more likely to sustain the use of an EBT.

\section{Method}

\section{Participants}

All eligible licensed psychiatric outpatient organizations from Pennsylvania $(n=508)$ were recruited to participate in the current study. Organizations that functioned across multiple counties, did not treat young children, had prior PCIT training experience, or a restricted service population were excluded. County administrators from each county were then approached to 
attend a required informational meeting about the study. Of the 94 organizations eligible to participate, 50 enrolled in the study.

Within each enrolled organization, one administrator, one supervisor, and two clinicians were invited to participate in the study. Administrators eligible to participate had to be employed as an Executive Director, Chief Financial Officer, or be responsible for the organization's daily operations. In order for clinicians to be eligible to participate, they had to: (a) hold a master's or doctoral-level degree, (b) be licensed or license-eligible, (c) see a caseload of appropriate PCIT families, (d) have no former PCIT training, and (e) be willing to complete various study tasks. Clinicians and administrators were still able to participate in the study if they left their organization over the course of the study.

Participants included 50 administrators and 100 clinicians from 50 organizations across Pennsylvania (PI: Herschell; NIMH RO1 MH095750). Participant characteristics are displayed in Table 1.

\section{Procedure}

Design. Enrolled organizations were randomized at the county-level to one of three training conditions. Randomization was balanced based on two covariates (i.e., population size and poverty level), comparable to prior studies that also utilized county-level randomized controlled designs (Aarons et al., 2009; Chamberlain et al., 2008; Wang, Saldana, Brown \& Chamberlain, 2010).

Training. The three training conditions included the cascading model (CM), distanceeducation (DE), and learning collaborative (LC). The CM, commonly referred to as a "Train-theTrainer" model, is a hierarchical model that involves training one clinician in an organization, who is then able to train other clinicians within their organization. Clinicians in the CM model 
attended a 40-hour workshop, followed by 16-hours of advanced training 6-months later. The CM model aligns with PCIT International's Training Guidelines and emphasizes clinician fidelity to the treatment. The DE condition, a web-based training model, involved clinicians completing a 10-hour online course (University of California, Davis, 2014). The LC model, originally developed by the Institute for Health Improvement, involves training multiple levels within an organization. Clinicians in the LC model completed three 2-day workshops over the course of 12 months. Within the LC model, core teams are organized within each organization and cross-site sharing among teams is encouraged. The LC model promotes developing a learning organization that is prepared to implement an intervention.

Following training completion, clinicians attended up to 24 1-hour consultation calls over the course of a year. Trainers recommended that clinicians attend $80 \%(n=20)$ of all consultation calls. Trainers with expertise in PCIT and disruptive behaviors conducted training and consultation calls and completed a training fidelity checklist at each training. Trainers were balanced across training condition and consultation call groups. Training occurred in four waves across Pennsylvania from 2012 through 2015.

Treatment. Parent-Child Interaction Therapy (PCIT; Eyberg \& Funderburk, 2011; McNeil \& Hembree-Kigin, 2010) is an EBT for children ages 2.5 to 7-years-old presenting with disruptive behaviors (e.g., oppositional defiance, noncompliance). PCIT has two phases of treatment. The Child-Directed Interaction phase focuses on improving the parent-child relationship. The second phase of treatment, Parent-Directed Interaction, involves teaching developmentally appropriate discipline skills (e.g., effective commands, time-out) to increase compliance. 
Compensation. Participants were compensated for participation in the study. Clinicians received free training, Continued Education Credits, and a monetary incentive for completion of study assessments ( $\$ 25$ for baseline and 6-months, \$30 for 12-months, and \$40 for 24-months). Organizations received free PCIT training for their participating clinicians as well as an initial stipend (\$1000) to cover equipment necessary for providing PCIT within their organization. All study procedures were approved by the University of Pittsburgh's and West Virginia University's Institutional Review Boards.

\section{Measures}

\section{Outcome.}

Sustainability. Clinician sustainability was measured according to three items from the Treatment Implementation Feedback Form and Your Experience-Treatment forms at 24-months. Clinicians provided an open-ended response to "Approximately what percentage of your caseload have you used PCIT with?" and "How many families have you yourself provided PCIT for since beginning training?" Clinicians also reported, "How much of the standard PCIT protocol are you still using?" with response options ranging from "Full," "Parts of it," and "I am not using it any longer."

Agency administrators reported on the sustainability of PCIT within their organization by completing three items from the EBP Sustaining Telephone Survey and Implementation Condition Feedback Form at 24-months. Administrators answered an open-ended question “Approximately what percentage of young children (aged 2.5 to 7) referred to your agency received PCIT in the last 6 months?" Administrators also responded to a dichotomous question, “Are you still offering PCIT" with response options of yes or no. Administrators completed a third question that asked, "Are you serving clients with this practice compared to the end 
of the project" with response options including: "a considerably fewer," "about the same," "a lot more.”

\section{Predictors.}

Clinician satisfaction. Clinicians described their satisfaction with providing PCIT at 12months on the Treatment Implementation Feedback Form. Clinicians were asked "How satisfied are you with PCIT?" with response options ranging from "Very dissatisfied" to "Very satisfied" on a 5-point Likert-type scale.

Organizational characteristics. Clinicians completed the Texas Christian UniversitySurvey of Organizational Functioning (TCU-SOF; Lehman, Greener, \& Simpson, 2002) at baseline and 12-months to measure organizational characteristics. The TCU-SOF is a 162-item self-report 5-point Likert-type scale with responses ranging from "Strongly Disagree" to “Strongly Agree.” It includes seven work climate domains: Motivation for Change, Resources, Staff Attributes, Organizational Climate, Job Attitudes, Workplace Practices, and Training Exposure and Utilization. The Resources, Organizational Climate, and Training Exposure and Utilization domains were used in the current study. Internal reliability estimates on these three scales range from .82 to .90 (Saldana et al., 2007). Within the current study, the SOF demonstrated excellent reliability across all subscales $(\alpha=.79-.89)$.

Training and consultation. Training condition (e.g., distance education, cascading model, learning collaborative) was included as a categorical variable that was dummy-coded. Trainers documented clinician consultation call attendance at each consultation call through the PCIT Case Consultation Outline \& Record. Clinicians could attend up to a total of 24 consultation calls.

\section{Data Analysis}


All analyses were conducted in Statistical Package for the Social Sciences or Mplus. To examine aim one, rates of sustainability of PCIT according to administrators and clinicians, descriptive statistics (e.g., means, correlations) were conducted on sustainability outcomes for clinicians and administrators.

For aims two and three, multi-level structural equation modeling with full information maximum likelihood using Mplus was utilized. Due to the level of missing data within the dataset, auxiliary variables that predicted missingness were included within the structural equation models. The data included clinicians nested within organizations; therefore, organization was utilized as a clustering variable in aims two and three. Sustainability was measured as a latent variable using the three individual items from clinicians reported at 24months.

First, a confirmatory factor analysis (CFA) was conducted to assess measurement fit of latent variables. Model fit was assessed using the $\chi^{2} / \mathrm{df}$ ratio $(\mathrm{CMIN})$ with values less than three demonstrating good fit, comparative fit index (CFI) with values above .95 signifying good fit, Tucker-Lewis Coefficient (TLI), with values close to 1.0 indicating good fit, and root-meansquare-error of approximation (RMSEA) with values less than .05 signifying good fit.

The proposed model for aim two, the impact of baseline organizational variables on sustainability, is displayed in Figure 1. The Resources, Organizational Climate, and Training Exposure \& Utilization domains from the SOF were utilized as latent variables predicting sustainability in this model. The Resources latent variable was created using the Offices, Staffing, and Training scales as these scales are most applicable to implementation rather than the Computer Access and e-Communications scales. A latent variable for the Organizational Climate latent variable included the Mission, Cohesion, and Change scales which align most 
centrally to the definition of organizational climate. The Training Exposure and Utilization latent variable included the Training Satisfaction, Training Exposure, and Training UtilizationIndividual Level scales. Three scales were chosen from each domain instead of all possible scales, due to limited power. Figure 2 displays the proposed structural equation model for aim three, examining the indirect effect of training design and consultation call attendance on sustainability through organizational resources, climate, and training exposure and utilization. Due to level of missing data and a lack of power, which is explained in detail below, observed variables of the Sustainability, Organizational Resources, Organizational Climate, Training Exposure and Utilization scales were utilized.

\section{Results}

\section{Missing Data}

Preliminary analyses were conducted to determine the amount of missing data, as well as patterns of missingness. On baseline organizational variables, the Resources and Organizational Climate scales had 0\% missing data, while the Training Exposure and Utilization scale had 2.0\% missing data. Missingness on each sustainability question (24-months) ranged from 39.0-55.0\% of cases. Little's MCAR test was statistically significant $(p=.044)$, indicating data was not missing completely at random. Separate variance t-tests were conducted to determine patterns of missing data, and if variances differed between cases with missing data and those with valid data. There were significant t-tests for the number of families enrolled in PCIT and number of EBTs used at baseline as predictors of missingness; therefore, these variables were included within the structural equation models as auxiliary variables to strengthen the robustness of measurement.

\section{Nesting of Data}


Several analyses were conducted to determine if nesting should be accounted for in the models. First, intraclass correlation coefficients (ICC's) were calculated with organization as the between-groups clustering variable. The ICC's ranged from .02 to .16 on sustainability outcomes. Second, the analyses for aims two and three were conducted with and without accounting for nesting of clinicians within organizations and changes in model fit were observed. For aim two, including organization as a clustering variable did not impact model fit $(\Delta \mathrm{CFI}=$ $.00, \triangle \mathrm{RMSEA}=.00)$. For aim three, the inclusion of organization as a clustering variable resulted in improved model fit $(\triangle \mathrm{CFI}=.08, \Delta \mathrm{RMSEA}=.07)$.

\section{Descriptive Statistics}

A majority of clinicians $(n=69 ; 77 \%)$ participated in at least $70 \%$ of all training hours in their training condition. Across all conditions, clinicians participated in an average of 15.64 (SD $=7.79)$ consultation calls. Clinicians reported on average feeling "somewhat satisfied" ( $M=$ 4.24, $S D=.95$ ) with PCIT at 12-months. Clinicians reported their perceptions of organizational resources $(M=34.16, S D=5.52)$, organizational climate $(M=34.53, S D=4.90)$, and training exposure and utilization $(M=31.83, S D=6.19)$. Clinician responses on the Organizational Climate scale are comparable to published norms $(M=33.9)$ available for this scale (Institute of Behavioral Research, 2005). There were moderate positive correlations between baseline organizational climate and training exposure and utilization $(r(100)=.54, p<.001)$, organizational climate and resources $(r(100)=.73, p<.001)$, and resources and training exposure and utilization $(r(100)=.62, p<.001)$.

Chi-square tests of independence were conducted to determine if there were any differences between training conditions on completion of training activities. Clinicians in the CM condition were more likely to complete at least $70 \%$ of their training hours, $\chi 2(3,90)=15.15, p$ 
$=.001$. There were no significant differences in attendance of consultation calls by condition, $\chi 2$ $(3,96)=55.59, p=.113$.

Chi-square tests of independence were conducted on sustainability outcomes and baseline organizational variables. There were no significant differences between training conditions on baseline organizational variables. The chi-square test of independence was significant for clinician reported use of the standard PCIT protocol, $\chi 2(3,56)=13.60, p=.01$. Clinicians in the CM condition were less likely to still be using the standard PCIT protocol in comparison to clinicians in the LC or DE conditions. All other chi-square tests of independence on sustainability outcomes were non-significant.

\section{Rates of Sustainability}

Administrators and clinicians reported the extent to which they were still using PCIT within their organization at 24-months (12-months post-training). A majority $(n=37 ; 74 \%)$ of administrators reported they were still offering PCIT at their organization at 24-months. Administrators stated approximately $10 \%(S D=17.31)$ of young children referred to their agency received PCIT in the last 6 months. Most administrators $(n=21 ; 57 \%)$ noted their organization was serving about the same number of clients appropriate for PCIT at 24-months as when training ended. Eight (22\%) administrators indicated their organization was serving considerably fewer clients, while eight (22\%) other administrators reported their organization was serving considerably more clients. Several administrators $(n=6)$ reported that although their organization was still offering PCIT, they had not served any families with PCIT in the past year.

On average, clinicians delivered PCIT to 8.31 families $(S D=7.43)$ and graduated (i.e., completed the entire PCIT protocol) $1.32(S D=1.70)$ families since the beginning of training. Clinicians reported using PCIT with approximately $16 \%(S D=21.58)$ of their caseload of young 
children at 24-months. At 24-months, 73\% $(n=41)$ of clinicians who responded reported they were still using the full PCIT protocol, while 18\% $(n=10)$ of clinicians were using parts of it, and $9 \%(n=5)$ were no longer using it. Correlations between clinician- and administrator- report of sustainability within their organization are displayed in Table 2 . There was a moderate positive correlation between administrator and clinicians report of the percentage of eligible children referred to their organization that received PCIT, $r(49)=.44, p=.006$.

\section{Measurement of Latent Variables}

Prior to running aims two and three, a CFA was conducted to examine the measurement fit of all latent variables. A CFA was conducted in which all four latent variables (sustainability, organizational climate, training exposure and utilization, and resources) were allowed to covary. The resulting model fit was poor, $\chi^{2}=182.83, p<.001, \mathrm{CFI}=.84, \mathrm{TLI}=.77$, and $\mathrm{RMSEA}=.17$. The CFA also was positive definite, indicating either inadequate power or correlations between variables greater than or equal to one. The modification indices suggested covarying the residual variances between the cohesion observed variable and the staff observed variable. This modification was made to the model, but did not result in greatly improved model fit, $\chi^{2}=$ $881.22, p<.001, \mathrm{CFI}=.87, \mathrm{TLI}=.81$, and $\mathrm{RMSEA}=.15$.

In order to determine if the issues with model fit were due to highly correlated predictor variables or sample size, several analyses were conducted. First, three CFAs were run to determine if the correlations greater than or equal to one were due primarily to one of the three organizational predictor latent variables. A CFA including organizational climate and sustainability was conducted, which resulted in correlations almost equal to one and poor model fit, $\chi^{2}=198.69, p<.001, \mathrm{CFI}=.79, \mathrm{TLI}=.61$, and $\mathrm{RMSEA}=.22 . \mathrm{A}$ CFA including organizational resources and sustainability resulted in correlations almost equal to one and poor 
model fit, $\chi^{2}=76.25, p<.001, \mathrm{CFI}=.71, \mathrm{TLI}=.46$, and $\mathrm{RMSEA}=.29$. A CFA including training exposure and utilization resulted in correlations almost equal to one and adequate model fit, $\chi^{2}=201.22, p<.001, \mathrm{CFI}=.96, \mathrm{TLI}=.93$, and RMSEA $=.09$. Finally, a CFA was conducted which included all nine observed scales as one organizational latent variable which was covaried with sustainability. This CFA resulted in correlations greater than or equal to one and poor model fit, $\chi^{2}=881.22, p<.001, \mathrm{CFI}=.86, \mathrm{TLI}=.81$, and $\mathrm{RMSEA}=.16$. Given that the issues with correlations were present across all of the CFAs and that the organizational predictor variables were not highly correlated $(>.80)$ in Pearson correlations, the issues in model fit were most likely due to inadequate power. Therefore, observed variables of the organizational predictor variables were utilized to increase statistical power.

\section{Organizational Barriers and Facilitators to Sustainability}

Model fit. Aim two examined the impact of baseline organizational variables on clinical sustainability of PCIT at 24-months. Figure 1 displays the proposed structural model for baseline organizational variables predicting sustainability, with clinician satisfaction with PCIT at 12months as a covariate. The organizational variables were allowed to covary given their moderately high correlations. The structural model resulted in poor fit, $\chi^{2}=48.89, p<.001, \mathrm{CFI}$ $=.01, \mathrm{TLI}=-1.18$, and RMSEA $=.26$. Given that model fit was poor with sustainability as a latent variable, the three individual indicators of sustainability were used as observed variables.

The model only including observed variables resulted in poor model fit, $\chi^{2}=163.50, p<$ $.001, \mathrm{CFI}=.00, \mathrm{TLI}=-15.64$, and $\mathrm{RMSEA}=.73$. The modification indices suggested covarying 24-months protocol use and approximate caseload. This modification improved model fit although it was still poor: $\chi^{2}=123.23, p<001, \mathrm{CFI}=.00, \mathrm{TLI}=-14.00$, and $\mathrm{RMSEA}=.69$. Satisfaction was removed from the model as a covariate given that it did not significantly predict 
sustainability outcomes, resulting in good model fit: $\chi^{2}=.26, p=.88, \mathrm{CFI}=1.00$ and $\mathrm{RMSEA}=$ .00 .

Associations between organizational variables and sustainability. The final model including standardized estimates is displayed in Figure 3. Results indicated that greater training exposure and utilization at baseline significantly predicted having a greater PCIT caseload at 24months $(\beta=.60, \mathrm{SE}=.15, p<.001)$; however, it predicted delivering PCIT to a fewer number of cases since the beginning of training $(\beta=-.38, \mathrm{SE}=.12, p<.001)$ and being less likely to still be using the full PCIT protocol $(\beta=.45, \mathrm{SE}=.12, p=.01)$. Baseline organizational climate and organizational resources did not significantly predict approximate caseload, use of PCIT protocol, or number of families seen since the beginning of training at 24-months. The overall model accounted for $37 \%$ of the variance in PCIT caseload at 24-months, 34\% of the variance in number of families delivered PCIT to since the beginning of training, and 19\% of a clinician's use of the PCIT protocol.

\section{Effects of Training and Consultation on Sustainability}

Model fit. Aim three focused on identifying the direct and indirect effects of training condition and consultation on sustainability (see Figure 2). All three sustainability outcomes (24months) were regressed onto training condition and consultation call attendance. Indirect paths from training condition and consultation call attendance through training exposure and utilization, resources, and organizational climate at 12-months to sustainability at 24-months were also created. Satisfaction with PCIT at 12-months was included as a covariate that predicted sustainability outcomes at 24 -months. The resulting model had poor fit: $\chi^{2}=159.08, p$ $<.001, \mathrm{CFI}=.89, \mathrm{TLI}=.53$, and $\mathrm{RMSEA}=.14$. Satisfaction was removed as a covariate given that it did not significantly predict any outcomes. The removal of satisfaction from the model 
resulted in good model fit: $\chi^{2}=132.66, p<.001, \mathrm{CFI}=.99, \mathrm{TLI}=.87$, and $\mathrm{RMSEA}=.07$. The final model is displayed in Figure 4.

Associations among organizational variables and training. Figure 5 displays the associations among organizational variables, training condition, and consultation calls. Clinicians in the CM condition relative to the $\mathrm{DE}$ condition $(\beta=.42, \mathrm{SE}=.14, p=.002)$ rated their organizations as having a greater amount of resources at 12-months. Clinicians in the CM condition also reported more training exposure and utilization $(\beta=.71, \mathrm{SE}=.07, p<.001)$ and a more positive organizational climate $(\beta=.65, \mathrm{SE}=.11, p<.001)$ at 12 -months relative to the $\mathrm{DE}$ condition. Clinicians in the LC model reported a more negative organizational climate $(\beta=-.30$, $\mathrm{SE}=.11, p=.003)$ and fewer organizational resources $(\beta=-.29, \mathrm{SE}=.09, p=.002)$ in comparison to the DE model. Clinicians who attended a greater number of consultation calls rated their organizations as having more resources $(\beta=.52, \mathrm{SE}=.11, p<.001)$ but not more training exposure and utilization $(p=.38)$ or a more positive organizational climate $(p=.82)$ at 12-months.

More training exposure and utilization at 12-months was associated with a more positive organizational climate at 12 -months $(\beta=.36, \mathrm{SE}=.11, p=.001)$. Greater training exposure and utilization was associated with a greater PCIT caseload at 24-months $(\beta=.22, \mathrm{SE}=.11, p=.04)$. Having more resources at 12-months was also associated with having a greater PCIT caseload ( $\beta$ $=.34, \mathrm{SE}=.11, p=.001)$, delivering PCIT to more families since beginning training $(\beta=.63$, $\mathrm{SE}=.12, p<.001)$, and being less likely to use the full PCIT protocol at 24 -months $(\beta=.47, \mathrm{SE}$ $=.15, p=.002)$.

Direct effects of training and consultation. Figure 6 displays the direct effects of training condition and consultation calls on sustainability. Attending more consultation calls was 
significantly associated with being more likely to have delivered PCIT to a fewer number of families since beginning training $(\beta=-.25, \mathrm{SE}=.10, p=.009)$. It was not associated with having a greater number of PCIT families in a clinician's caseload $(\beta=.12, \mathrm{SE}=.37, p=.75)$ or using the full PCIT protocol at 24-months $(\beta=-.19, \mathrm{SE}=.20, p=.34)$.

Clinicians in the CM were more likely to have a higher average PCIT caseload at 24months than clinicians in the $\mathrm{DE}$ training condition $(\beta=.34, \mathrm{SE}=.15, p=.03)$. Clinicians in the LC condition did not differ significantly from clinicians in the DE training condition in average PCIT caseload at 24-months $(p=.79)$. Clinicians in the LC model were less likely to still be using the full PCIT protocol compared to clinicians in the DE training model $(\beta=.28, \mathrm{SE}=.11$, $p=.01)$. Clinicians in the CM model did not differ from clinicians in the DE training model on use of the PCIT protocol $(p=.70)$. Training conditions did not significantly differ in the number of families that received PCIT since beginning training.

Indirect effects. There were significant indirect effects of training condition on approximate PCIT caseload, number of families that received PCIT since beginning training, and clinician use of the PCIT protocol at 24-months. The total indirect effect for the CM condition relative to the DE condition on PCIT caseload at 24-months was .26 $(S E=.12, p=.03)$. Clinicians in the CM condition had a higher PCIT caseload at 24-months, through more resources $(\beta=.14, \mathrm{SE}=.06, p=.02)$. The total indirect effect for the $\mathrm{CM}$ condition relative to the DE condition on number of families receiving PCIT since beginning training was $.42(S E=$ $.12, p=.001)$. Clinicians in the CM condition delivered PCIT to more families since beginning training through greater resources at 12 -months compared to the DE condition $(\beta=.27, \mathrm{SE}=.01$, $p=.006)$. The total indirect effect for the CM condition on use of the PCIT protocol was .25 (SE 
$=.10, p=.013)$. Clinicians in the CM condition were less likely to be using the full PCIT protocol at 24-months due to having more organizational resources $(\beta=.19, \mathrm{SE}=.07, p=.009)$.

Consultation calls had a significant total indirect effect on approximate caseload $(\beta=.32$, $\mathrm{SE}=.12, p<.001)$, number of families receiving PCIT since beginning training $(\beta=.54, \mathrm{SE}=$ $.17, p=.002)$, and use of the PCIT protocol $(\beta=.37, \mathrm{SE}=.15, p=.01)$. There was a specific indirect effect of consultation on these sustainability outcomes throughout organizational resources. There were specific indirect effects of consultation through organizational resources on approximate PCIT caseload $(\beta=.18, \mathrm{SE}=.07, p=.01)$, number of families that received PCIT since beginning training $(\beta=.33, \mathrm{SE}=.11, p=.003)$, and use of the PCIT protocol $(\beta=$ $.26, \mathrm{SE}=.11, p=.02)$.

The overall model accounted for $42 \%$ of the variance in approximate PCIT caseload, $58 \%$ of the variance in number of families receiving PCIT since beginning training, and 35\% of the variance in use of the PCIT protocol. Forty percent of the variance in resources, $30 \%$ of the variance in organizational climate, and $50 \%$ of the variance in training exposure and utilization was explained by the model.

\section{Discussion}

The purpose of this study was to gain an in-depth understanding of the sustainability of PCIT following a statewide training initiative. This study was one of the first, to our knowledge, to examine the sustainability of PCIT, and to utilize both administrator- and clinician-reports. This study had three primary aims: (1) to describe rates of sustainability for clinicians and administrators, (2) to identify organizational variables which impact sustainability, and (3) to understand the direct and indirect influence of training and consultation on sustainability. Results 
from this study have important implications for increasing access to EBTs within community settings.

\section{Rates of Sustainability}

The first aim of this study was to describe the extent to which clinicians and administrators were still using PCIT at 24-months (or one year after the completion of the implementation phase). A majority of clinicians and administrators reported they were still offering or providing PCIT and were delivering PCIT to a similar number of clients as when training ended. The rates of sustainability within the current study were comparable to previous studies examining sustainability at the same time-point (Bond et al., 2014; Cooper et al., 2015; Scheirer, 2005). Given that this study measured sustainability one year after the completion of the implementation phase, it is possible that fewer organizations will still be utilizing PCIT after a longer time-period as rates of sustainability decrease over time (Brookman-Frazee et al., 2016). Additionally, there were small to moderate correlations between clinician and administrator reports of sustainability. These low rates of concordance may be due to actual differences in sustainability within organizations. For example, while an administrator may report that PCIT is still being offered as an intervention, clinicians within the same organization may not be delivering PCIT often. On the other hand, this result may reveal inconsistencies in the reporting of sustainability. Therefore, future studies should attempt to identify the most reliable and accurate reporters of sustainability. Most clinicians also indicated they were still using all parts of the PCIT protocol, which may suggest that clinicians believed that they were able to maintain fidelity to the treatment protocol after training has ended. This finding should be interpreted with caution as it is based on self-report and it has been established that there is often a discrepancy between behavioral observations and self-report measures (Hogue et al., 2015). 


\section{Organizational Barriers and Facilitators}

A second aim of the study was to identify baseline organizational barriers and facilitators to sustainability. Previous literature has supported that organizational factors have a significant influence on sustainability, and thus they were chosen as the focus of the current study (Cooper et al., 2015; Stirman et al., 2012). The hypotheses of this aim were partially supported. This study found that greater training exposure and utilization at baseline predicted using the full PCIT protocol and having a greater caseload of PCIT clients at 24-months. This finding is similar to previous research which suggests that training initiatives can be helpful to the sustainability of an EBT (Aarons et al., 2009). Previous research has shown that organizations which are already providing opportunities for training appear to be more likely to sustain the use of an intervention. In contrast, this study found that clinicians with more training exposure and utilization delivered PCIT to a fewer number of clients since the beginning of training. During training and initial consultation calls, clinicians are often encouraged to see a high number of cases initially due to attrition (Jackson et al., 2017). While some clinicians may initially see a large number of PCIT clients due to these recommendations, they may not continue to sustain a high PCIT caseload. This finding may also be a result of measurement error, due to the fact that this question measured the total number of clients since the beginning of training, instead of the number of clients being seen at 24-months. Therefore, this number may not accurately reflect sustainability and may be a better indicator of initial implementation.

In contrast to previous findings, having a more positive organizational climate at baseline did not predict sustainability (Lyon et al., 2011). This finding was surprising as it was expected that organizations which shared a collective mission and values, particularly in support of PCIT, would be more likely to sustain its use. On the other hand, this suggests that despite having a 
negative organizational climate, clinicians may still be able to deliver EBTs if they receive adequate training in their use. Additionally, recent research has suggested that aspects of an organization's climate may interact in ways that affect sustainability. Williams and colleagues (2018) found that a positive organizational climate at baseline interacts with a positive implementation climate, thereby increasing sustainability. Therefore, the presence of other organizational characteristics in addition to a positive organizational climate may be necessary to produce long-term use of a treatment.

Finally, baseline organizational resources (e.g., office space, adequate staffing) did not predict sustainability, although resources at 12-months did predict sustainability. This contrasts with previous findings that greater initial administrative resources are associated with greater sustainability (Loman et al., 2010; Tibbits et al., 2010). This finding suggests organizational patterns predict sustainability at different time-points, and it is important to consider these dynamic changes in research. For example, adequate initial funding has been suggested to be an important resource that promotes sustainability (Cooper et al., 2015). It is possible that some resources, particularly financial, are important early on for sustainability; however, the resources measured within the current study focused primarily on the availability of office space and administrative staff. There has been research previously that supports the effectiveness of organizational resources in predicting sustainability, yet there is limited research on what type of resources are important (Cooper et al., 2015; Stirman et al., 2012). Future research should continue to identify the aspects of organizational resources which are most likely to promote sustainability.

Overall, this aim revealed that organizational characteristics had various impacts on sustainability, with a majority of organizational facets not being associated with sustainability. 
Given that many organizational characteristics did not facilitate sustainability, it is possible that other factors are more important to consider. For example, issues such as the number of referrals an organization receives for PCIT-appropriate clients may have impacted these findings.

Although all organizations within the study received PCIT-appropriate referrals, there was likely considerable variability in the total number of referrals received by each organization, and also by clinicians within each organization. Future research should attempt to balance or match organizations and clinicians on caseload characteristics to obtain a more comprehensive understanding of sustainability. Additionally, it is possible that factors at the clinician-level may have more of an impact on sustainability. Although organizations may be impacted by issues such as referrals, clinicians may have more power and ability than organizations to choose what treatments their clients receive. Interestingly, clinician satisfaction with PCIT was not associated with sustainability. This finding may partially be related to the lack of variability in clinician satisfaction with PCIT within the current study, given that most clinicians were very satisfied with PCIT. Therefore, further research should examine these client-level factors that impact sustainability.

\section{Training Condition and Consultation}

The third aim of this study focused on the direct and indirect effects of training design and consultation on sustainability outcomes. Clinicians who attended more consultation calls delivered PCIT to a fewer number of clients since beginning training. Additionally, they did not have a higher PCIT caseload at 24-months and did not report fuller use of the PCIT protocol compared to clinicians who attended less calls. This contrasts with previous research suggesting the importance of consultation as a training component for promoting implementation and sustainability (Beidas et al., 2012; Jackson et al., 2017). Few empirical studies on consultation 
have examined this training component in relation to actual use of an intervention (Schoenwald et al., 2003). Studies have instead primarily focused on how consultation increases clinician knowledge, skill, and adherence to a treatment. Given that this study did not find that consultation calls had an effect on clinician reported use of PCIT, it is possible that other variables influence a clinician's use of the intervention. Another possible explanation is that clinicians who were able to attend more consultation calls had a lower overall caseload, were part-time, or contractors, and had more time to attend consultation calls. Clinicians who were full-time at their organization may have been unable to fit the consultation calls into their schedules, thus leading to lower consultation call attendance despite having a greater PCIT caseload. The current study did not measure this possibility, so it will be important to consider in future research.

Overall, utilizing a cascading training condition appears to have several strengths in comparison to learning collaborative or distance education approaches. Clinicians within the cascading training condition had more PCIT clients in their caseload at 24-months relative to clinicians in other conditions. This finding builds upon previous research suggesting that brief trainings are insufficient for producing changes in clinician behavior and adds to research by identifying specific training types that are related to increased sustainability (Herschell et al., 2010; Beidas et al., 2012). While there has been limited research on these training models, previous research has found that although this model may produce initial changes in behavior, they are often not maintained over time (Shore et al., 1995; Martino et al., 2010). In contrast, this study suggests that the cascading model can lead to sustained changes in clinician behavior over time, specifically related to the number of clients receiving an EBT. Further research is necessary 
to determine if this sustainability is related to improved client functioning (e.g., reductions in problem behaviors).

This study also found that clinicians in the learning collaborative condition were less likely to be using the full PCIT protocol at 24-months. Based upon these results, it is unclear if these clinicians are no longer using PCIT within their organization, or if they modified the PCIT protocol. If they were no longer using the PCIT protocol at all, it is unlikely that these organizations sustained the use of PCIT. On the other hand, if these clinicians are modifying the PCIT protocol based upon client needs, this may lead to greater sustainability given that clinicians who modify a treatment protocol may be more likely to sustain its use for greater periods of time (Stirman et al., 2012). It is important to consider, however, that the extent to which treatment protocols are adapted may alter the efficacy of these interventions although this has not yet been tested.

Clinicians in the cascading condition also reported more positive organizational characteristics following training. They perceived their organizations as having a more positive organizational climate, resources, and training exposure and utilization following training. Many aspects of the cascading condition lend itself to changing organizational characteristics. This training design involves training clinicians within the organization to continue facilitating clinician knowledge and use of an intervention, while also allowing other clinicians within the organization to be trained. By developing these resources within the organization, clinicians may feel more supported and prepared to utilize an intervention. Organizational trainings, such as the Availability, Responsiveness, and Continuity (ARC) Organizational Intervention have been developed to improve the organizational social context of mental health agencies (Glisson \& Schoenwald, 2005; Williams et al., 2017). These trainings often involve focusing on improving 
communication between administrators and clinicians, and reducing barriers to effective service within an organization. It is possible that the process of training senior clinicians with an organization in the cascading training model also serves this same purpose. Interestingly, clinicians do not frequently train other clinicians within their organization after they have been trained as expert trainers. Due to this finding, it is more likely that having a clinician within the organization serves the function as a support system for the clinicians, rather than actual trainers to provide ongoing training. This suggests that an important facet of effective training is increasing supports available within an organization to continue to provide PCIT.

It is surprising that clinicians in the $\mathrm{LC}$ condition did not also endorse positive perceptions of their organization. In contrast, clinicians in the LC condition rated their organizations as having a more negative organizational climate, fewer resources, and less training exposure and utilization. This finding was unexpected, given that a unique feature of the LC model is its focus on creating learning organizations, and training multiple levels within an organization. Based upon anecdotal evidence from training satisfaction surveys, there are several possibilities as to why clinicians in this model perceived their organizations negatively. One explanation for this finding is given that clinicians, supervisors, and administrators were all trained in this condition, clinicians may have felt that their supervisors and administrators were too involved in their clinical practice. The LC training model also included several organizations at each training; therefore, clinicians were able to compare aspects of their organization to other organizations. Clinicians may have then viewed other organizations within the training as having more resources and opportunities, leading to a more negative view of their own organization. Further research is necessary to understand the causes of this finding, particularly as this training 
model continues to be required by organizations such as the National Traumatic Stress Network to disseminate EBTs.

This study sought to identify mechanisms of change through which training and consultation influence sustainability. It appears that the positive effects of the cascading training condition influenced sustainability primarily through increasing organizational resources and training exposure. Increasing organizational resources may be particularly important for interventions such as PCIT, which require longer sessions and specialized equipment (e.g., bugin-the-ear device) to conduct treatment. Interestingly, the cascading condition was associated with greater resources and being less likely to deliver the full PCIT protocol. This may be similar to other findings within the study that suggest clinicians are more likely to sustain an intervention when they can flexibly adapt it (Stirman et al., 2012). Additionally, clinicians in the cascading condition reported higher relative training exposure and utilization, which was related to a greater PCIT caseload. Given that the cascading condition trains senior clinicians within an organization, other clinicians within the same organization may receive more training opportunities over time that increase their use of an intervention.

\section{Strengths}

This study has several notable strengths that enhance the current body of literature on sustainability. First, this study utilized multiple informants within an organization to understand sustainability. This contrasts with previous studies which have primarily relied on one question from administrators to determine an organization's sustainability (Stirman et al., 2012). Assessing sustainability through multiple reporters may help refine sustainability research, and have greater clinical implications to the field of implementation science. Similarly, another strength of this study was that it evaluated sustainability according to several indicators at the 
organizational- and client-level. While sustainability has been suggested to be a multi-faceted concept, very few studies have examined this (Scheirer, 2005). This complements existing research by strengthening the understanding of the measurement of sustainability. A third strength of this study was its focus on implementation mechanisms. This is an important step in understanding how implementation strategies work (Lewis et al., 2018). Previous studies have had difficulty identifying mechanisms of change. For example, in a review of nine implementation studies in mental health, none of the included studies supported a hypothesized mediator (Williams, 2016). This study is a promising step in understanding processes through which implementation works.

\section{Limitations}

Several limitations of the current study should also be taken into consideration. One of the most significant limitations to this study is the small sample size, which led to inadequate power to conduct several analyses. Although steps were taken to increase retention in the study (e.g., incentives), a large amount of missing data was present at 24-months. Additionally, the data were not missing completely at random, suggesting that individuals with missing data may have differed from those who completed all assessments. While the researchers attempted to reduce the effect of missingness through the use of Mplus and auxiliary variables, the results of the current study may have still been impacted by missingness. Similarly, latent variables were unable to be utilized in the current study due to the amount of missing data which resulted in poor model fit of latent variables. The use of latent variables would have strengthened this study due to its ability to increase the robustness of measurement of constructs and its ability to estimate measurement error. 
Another limitation of the current study was the reliance on self-report and studydeveloped measures. As has been noted previously, self-report measures may not accurately reflect a clinician's behavior (Hogue et al., 2015). Therefore, the conclusions drawn from the current study should be interpreted with caution as behavioral observations were not used to assess adherence to PCIT or use of the intervention. A similar concern is the use of studydeveloped measures which also lack psychometric evidence. This is a problem that is common to the field of implementation science due to the advancement of the field prior to the development of adequate measures (Martinez, Lewis, \& Weiner, 2014).

There were also limitations specific to the measurement of the sustainability variables. For example, a clinician's caseload at 24-months was measured as a percentage of their total caseload rather than the total number of PCIT clients. While this may have helped account for variation in the total number of clients seen by clinicians, it may have hindered the interpretation of this item. Similarly, a clinician's use of the PCIT protocol included response options of utilizing the full, some, or none of the protocol. This question may have been a more accurate representation of adherence to the PCIT protocol if it only included response options of modifications (e.g., some, most, full) rather than use (e.g., none) of the protocol.

Another limitation to the current study was the measurement of organizational variables. While the organizational measure utilizing has demonstrated psychometric evidence for its subscales, we were unable to examine all subscales due to a lack of power. Additionally, the Resources subscale within the SOF focused on technological resources, such as office space and computer access. Although these resources may be important to implementation, this scale did not capture other resources (e.g., financial) which may be equally as important. 
A final limitation to the current study includes the lack of variability in some of the sustainability outcomes. Within the current study, most clinicians who responded reported still using the full PCIT protocol. Therefore, the findings may have been impacted by having few clinicians that endorsed no longer using the PCIT protocol. Given the issues with missing data within the current study, it is also possible that the clinicians who did not respond to this item were no longer using the PCIT protocol. While missing data was accounted for through the use of Mplus and auxiliary variables, it is likely that these findings were still impacted by the amount of missing data.

\section{Future Directions}

Future research can build upon the current study in several ways. First, this is one of the first studies to identify organizational resources as a mechanism of change that facilitates sustainability. This serves as an important starting point for mechanisms research within implementation science; however, the study of additional mechanisms is important to consider within future research. This study primarily focused on organizational mechanisms of change, yet clinician- and family-level should also be a focus of future research. Second, this study's utilization of both administrator- and clinician-reports of sustainability suggests an important area of research. This study revealed that administrators and clinicians have moderate concordance in their reports of sustainability, and it is unclear whether this is due to the measurement of the construct or differences in reporting bias. Future research should attempt to disentangle this question, and identify which stakeholders are the most accurate reporters of sustainability and implementation outcomes. Importantly, structural equation modeling approaches should be utilized in future studies to reduce the impact of measurement error on these analyses. 


\section{Conclusions}

As there are increased efforts to disseminate and implement EBTs, implementation strategies must be refined to enhance the transportability of EBTs into community settings. Understanding factors that improve the sustainability of EBTs is particularly important to increase the public health impact of mental health interventions. Findings from this study suggest that organizational characteristics at baseline and during implementation, as well as training and consultation calls may significantly influence the extent to which a clinician sustains an intervention. Organizations seeking to train their clinicians should carefully consider the type of training they decide to pursue based upon their individual needs and goals for training. The type of training an organization is involved in may play a significant role in the number of families that ultimately have access to empirically supported treatments. 


\section{References}

Aarons, G. A., Wells, R. S., Zagursky, K., Fettes, D. L., \& Palinkas, L. A. (2009). Implementing evidence-based practice in community mental health agencies: A multiple stakeholder analysis. American Journal of Public Health, 99(11), 2087-2095. https://doi.org/10.2105/AJPH.2009.161711

Abraham, A. J., Knudsen, H. K., \& Roman, P. M. (2011). A longitudinal examination of alcohol pharmacology adoption in substance use disorder treatment programs: Patterns of sustainability and discontinuation. Journal of Studies on Alcohol and Drugs, 72(4), 669677.

American Diabetes Association. (2004). The breakthrough series: IHI's collaborative model for achieving breakthrough improvement. Diabetes Spectrum, 17(2), 97-101.

APA Presidential Task Force on Evidence-Based Practice. (2006). Evidence-based practice in psychology. The American Psychologist, 61(4), 271.

Beidas, R. S., Edmunds, J. M., Marcus, S. C., \& Kendall, P. C. (2012). Training and consultation to promote implementation of an empirically supported treatment: A randomized trial. Psychiatric Services, 63(7), 660-665. https://doi.org/10.1176/appi.ps.201100401

Beidas, R. S., \& Kendall, P. C. (2010). Training therapists in evidence-based practice: A critical review of studies from a systems-contextual perspective. Clinical Psychology: Science and Practice, 17(1), 1-30. https://doi.org/10.1111/j.1468-2850.2009.01187.x

Beidas, R. S., Edmunds, J., Ditty, M., Watkins, J., Walsh, L., Marcus, S., \& Kendall, P. (2014). Are inner context factors related to implementation outcomes in cognitive-behavioral therapy for youth anxiety?. Administration and Policy in Mental Health and Mental Health Services Research, 41(6), 788-799. 
Bond, G. R., Drake, R. E., McHugo, G. J., Peterson, A. E., Jones, A. M., \& Williams, J. (2014). Long-term sustainability of evidence-based practices in community mental health agencies. Administration and Policy in Mental Health and Mental Health Services Research, 41(2), 228-236. https://doi.org/10.1007/s10488-012-0461-5

Brookman-Frazee, L., Stadnick, N., Roesch, S., Regan, J., Barnett, M., Bando, L., ... Lau, A. (2016). Measuring sustainment of multiple practices fiscally mandated in children's mental health services. Administration and Policy in Mental Health and Mental Health Services Research, 43(6), 1009-1022. https://doi.org/10.1007/s10488-016-0731-8

Brownson, R. C., Kreuter, M. W., Arrington, B. A., \& True, W. R. (2002). Translating scientific discoveries into public health action: How can schools of public health move us forward? Public Health Reports, 117, 485-488. https://doi.org/10.1016/S0033-3549(04)50191-7

Bunger, A. C., Hanson, R. F., Doogan, N. J., Powell, B. J., Cao, Y., \& Dunn, J. (2016). Can learning collaboratives support implementation by rewiring professional networks? Administration and Policy in Mental Health and Mental Health Services Research, 43(1), 79-92. https://doi.org/10.1007/s10488-014-0621-x

Calder, R., Ainscough, T., Kimergard, A., Witton, J., \& Dyer, K. R. (2017). Online training for substance misuse workers: A systematic review. Drugs: Education, Prevention and Policy, $24,1-13$.

Chamberlain, P., Brown, C. H., Saldana, L., Reid, J., Wang, W., Marsenich, L., ... \& Bouwman, G. (2008). Engaging and recruiting counties in an experiment on implementing evidencebased practice in California. Administration and Policy in Mental Health and Mental Health Services Research, 35(4), 250-260.

Chambers, D. A., Glasgow, R. E., \& Stange, K. C. (2013). The dynamic sustainability 
framework: Addressing the paradox of sustainment amid ongoing change. Implementation Science, 8(1), 1-11. https://doi.org/10.1186/1748-5908-8-117

Cooper, B. R., Bumbarger, B. K., \& Moore, J. E. (2015). Sustaining evidence-based prevention programs: Correlates in a large-scale dissemination initiative. Prevention Science, 16(1), 145-157. https://doi.org/10.1007/s11121-013-0427-1

DiMeo, M. A., Moore, G. K., \& Lichtenstein, C. (2012). Relationship of evidence-based practice and treatments: a survey of community mental health providers. Journal of Community Psychology, 40(3), 341-357. https://doi.org/10.1002/jcop.20516

Ditty, M. S., Landes, S. J., Doyle, A., \& Beidas, R. S. (2015). It takes a village: A mixed method analysis of inner setting variables and dialectical behavior therapy implementation. Administration and Policy in Mental Health and Mental Health Services Research, 42(6), 672-681.

Dopp, A. R., Hanson, R. F., Saunders, B. E., Dismuke, C. E., \& Moreland, A. D. (2017). Community-based implementation of trauma-focused interventions for youth: Economic impact of the learning collaborative model. Psychological Services, 14(1), 57-65. https://doi.org/10.1037/ser0000131

Dorsey, S., McLaughlin, K. A., Kerns, S. E., Harrison, J. P., Lambert, H. K., Briggs, E. C., ... \& Amaya-Jackson, L. (2017). Evidence base update for psychosocial treatments for children and adolescents exposed to traumatic events. Journal of Clinical Child \& Adolescent Psychology, 46(3), 303-330. https://doi.org/10.1080/15374416.2016.1220309

Doyle, C., Howe, C., Woodcock, T., Myron, R., Phekoo, K., McNicholas, C., ... Bell, D. (2013). Making change last: Applying the NHS institute for innovation and improvement sustainability model to healthcare improvement. Implementation Science, 8(1), 1-10. 
https://doi.org/10.1186/1748-5908-8-127

Evans, S. W., Owens, J. S., \& Bunford, N. (2014). Evidence-based psychosocial treatments for children and adolescents with attention-deficit/hyperactivity disorder. Journal of Clinical Child \& Adolescent Psychology, 43(4), 527-551.

https://doi.org/10.1080/15374416.2013.850700

Eyberg, S. M., \& Funderburk, B. (2011). Parent-child interaction therapy protocol. Gainesville, FL: PCIT International.

Glisson, C., \& Schoenwald, S. K. (2005). The ARC organizational and community intervention strategy for implementing evidence-based children's mental health treatments. Mental Health Services Research, 7(4), 243-259.

Herschell, A. D., Kolko, D. J., Baumann, B. L., \& Davis, A. C. (2010). The role of therapist training in the implementation of psychosocial treatments: A review and critique with recommendations. Clinical Psychology Review, 30(4), 448-466. https://doi.org/10.1016/j.cpr.2010.02.005

Herschell, A. D., Kolko, D. J., Scudder, A. T., Taber-Thomas, S., Schaffner, K. F., Hiegel, S. A., ... Mrozowski, S. (2015). Protocol for a statewide randomized controlled trial to compare three training models for implementing an evidence-based treatment. Implementation Science, 10(1), 133. https://doi.org/10.1186/s13012-015-0324-z

Higa-McMillan, C. K., Francis, S. E., Rith-Najarian, L., \& Chorpita, B. F. (2016). Evidence base update: 50 years of research on treatment for child and adolescent anxiety. Journal of Clinical Child \& Adolescent Psychology, 45(2), 91-113.

Hoagwood, K., Burns, B. J., Kiser, L., Ringeisen, H., \& Schoenwald, S. K. (2001). Evidencebased practice in child and adolescent mental health services. Psychiatric Services, 52(9), 
1179-1189. https://doi.org/10.1176/appi.ps.52.9.1179

Hogue, A., Dauber, S., Henderson, C. E., \& Liddle, H. A. (2014). Reliability of therapist selfreport on treatment targets and focus in family-based intervention. Administration and Policy in Mental Health and Mental Health Services Research, 41(5), 697-705.

Institute of Behavioral Research. (2005). TCU Survey of Organizational Functioning (TCU SOF). Fort Worth: Texas Christian University, Institute of Behavioral Research. Available at ibr.tcu.edu

Jackson, C. B., Herschell, A. D., Schaffne, K. F., Turiano, N. A., \& McNeil, C. B. (2017). Training community-based clinicians in parent-child interaction therapy: The interaction between expert consultation and caseload. Professional Psychology: Research and Practice, 48(6), 481-489. https://doi.org/10.1037/pro0000149

Jackson, C. B., Quetsch, L. B., Brabson, L. A., \& Herschell, A. D. (2018). Web-based training methods for behavioral health providers: A systematic review. Administration and Policy in Mental Health and Mental Health Services Research. https://doi.org/10.1007/s10488-018$\underline{0847-0}$

Jensen-Doss, A., Hawley, K. M., Lopez, M., \& Osterberg, L. D. (2009). Using evidence-based treaments: The experiences of youth providers working under a mandate. Professional Psychology: Research and Practice, 40(4), 417-424.

Kaminski, J. W., \& Claussen, A. H. (2017). Evidence base update for psychosocial treatments for disruptive behaviors in children. Journal of Clinical Child \& Adolescent Psychology, $46(4), 477-499$.

Kazdin, A. E. (2017). Addressing the treatment gap: A key challenge for extending evidencebased psychosocial interventions. Behaviour Research and Therapy, 88, 7-18. 
Kazdin, A. E. (2018). Expanding mental health services through novel models of intervention delivery. Journal of Child Psychology and Psychiatry. Advance online publication.

Kazdin, A. E., \& Blase, S. L. (2011). Interventions and models of their delivery to reduce the Burden of mental illness: Reply to commentaries. Perspectives on Psychological Science, 6(5), 507-510. https://doi.org/10.1177/1745691611418241

Kolko, D. J., Baumann, B. L., Herschell, A. D., Hart, J. A., Holden, E. A., \& Wisniewski, S. R. (2012). Implementation of AF-CBT by community practitioners serving child welfare and mental health: a randomized trial. Child Maltreatment, 17(1), 32-46. https://doi.org/10.1177/1077559511427346

Kolko, D. J., Iselin, A. M. R., \& Gully, K. J. (2011). Evaluation of the sustainability and clinical outcome of Alternatives for Families: A Cognitive-Behavioral Therapy (AF-CBT) in a child protection center. Child Abuse and Neglect, 35(2), 105-116.

https://doi.org/10.1016/j.chiabu.2010.09.004

Lamontagne, M.-E., Swaine, B. R., Lavoie, A., \& Careau, E. (2011). Analysis of the strengths, weaknesses, opportunities and threats of the network form of organization of traumatic brain injury service delivery systems. Brain Injury, 25(12), 1188-97. https://doi.org/10.3109/02699052.2011.608211

LaPelle, N. R., Zapka, J., \& Ockene, J. K. (2006). Sustainability of public health programs: The example of tobacco treatment services in Massachusetts. American Journal of Public Health, 96(8), 1363-1369. https://doi.org/10.2105/AJPH.2005.067124

Lee, H., Hall, A., Nathan, N., Reilly, K. L., Seward, K., Williams, C. M., ... \& Wolfenden, L. (2018). Mechanisms of implementing public health interventions: a pooled causal mediation analysis of randomised trials. Implementation Science, 13(1), 42 
Lehman, W. E., Greener, J. M., \& Simpson, O. D. (2002). Assessing organizational readiness for change. Journal of Substance Abuse Treatment, 22(4), 197-209. https://doi.org/10.1016/S0740-5472(02)00233-7

Lewis, C. C., Klasnja, P., Powell, B., Tuzzio, L., Jones, S., Walsh-Bailey, C., \& Weiner, B. (2018). From classification to causality: Advancing Understanding of Mechanisms of change in implementation science. Frontiers in Public Health, 6, 136.

Little, T. D. (2013). Longitudinal structural equation modeling. Guilford Press.

Loman, S. L., Rodriguez, B. J., \& Horner, R. H. (2010). Sustainability of a targeted intervention package: First step to success in Oregon. Journal of Emotional and Behavioral Disorders, $18(3), 178-191$.

Lyon, A. R., Frazier, S. L., Mehta, T., Atkins, M. S., \& Weisbach, J. (2011). Easier said than done: Intervention sustainability in an urban after-school program. Administration and Policy in Mental Health and Mental Health Services Research, 38(6), 504-517. https://doi.org/10.1007/s10488-011-0339-y

Markiewicz, J., Ebert, L., Ling, D., Amaya-Jackson, L., \& Kisiel, C. (2006). Learning collaborative toolkit. Los Angeles, California: National Center for Child Traumatic Stress.

Martinez, R. G., Lewis, C. C., \& Weiner, B. J. (2014). Instrumentation issues in implementation science. Implementation Science, 9, 118. https://doi.org/10.1186/s13012-014-0118-8

Martino, S., Ball, S. A., Nich, C., Canning-Ball, M., Rounsaville, B. J., \& Carroll, K. M. (2011). Teaching community program clinicians motivational interviewing using expert and trainthe-trainer strategies. Addiction, 106(2), 428-441. https://doi.org/10.1111/j.13600443.2010.03135.x

Martino, S., Brigham, G. S., Higgins, C., Gallon, S., Freese, T. E., Albright, L. M., ... Condon, 
T. P. (2010). Partnerships and pathways of dissemination: The National Institute on Drug Abuse-Substance Abuse and Mental Health Services Administration Blending Initiative in the Clinical Trials Network. Journal of Substance Abuse Treatment, 38(Suppl 1), S31-S43. https://doi.org/10.1016/j.jsat.2009.12.013

McIntosh, K., Kim, J., Mercer, S. H., Strickland-Cohen, K., \& Horner, R. H. (2014). Variables associated with enhanced sustainability of school-wide positive behavioral interventions and supports. Assessment for Effective Intervention, 40(3), 184-191.

McLennan, J. D., Wathen, C. N., MacMillan, H. L., \& Lavis, J. N. (2006). Research-practice gaps in child mental health. Journal of the American Academy of Child and Adolescent Psychiatry, 45(6), 658-665. https://doi.org/10.1097/01.chi.0000215153.99517.80

McMillen, J. C., Hawley, K. M., \& Proctor, E. K. (2016). Mental health clinicians' participation in web-based training for an evidence supported intervention: Signs of encouragement and trouble ahead. Administration and Policy in Mental Health and Mental Health Services Research, 43(4), 592-603. https://doi.org/10.1007/s10488-015-0645-x

McNeil, C.B., \& Hembree-Kigin, T.L. (2010). Parent-child interaction therapy. New York, NY: Springer Science \& Business Media.

Nadeem, E., Gleacher, A., \& Beidas, R. S. (2013). Consultation as an implementation strategy for evidence-based practices across multiple contexts: Unpacking the black box. Administration and Policy in Mental Health and Mental Health Services Research, 40(6), 439-450. https://doi.org/10.1007/s10488-013-0502-8

Palinkas, L. A., Weisz, J. R., Chorpita, B. F., Levine, B., Garland, A. F., Hoagwood, K. E., \& Landsverk, J. (2013). Continued use of evidence-based treatments after a randomized controlled effectiveness trial: A qualitative study. Psychiatric Services, 64(11), 1110-1118. 
https://doi.org/10.1176/appi.ps.004682012

Perou, R., Bitsko, R. H., Blumberg, S. J., Pastor, P., Ghandour, R. M., Gfroerer, J. C., ... \& Parks, S. E. (2013). Mental health surveillance among children-United States, 2005-2011. Morbidity and Mortality Weekly Report Surveillence Summary, 62(Suppl 2), 1-35.

Polanczyk, G. V., Salum, G. A., Sugaya, L. S., Caye, A., \& Rohde, L. A. (2015). Annual research review: A meta-analysis of the worldwide prevalence of mental disorders in children and adolescents. Journal of Child Psychology and Psychiatry and Allied Disciplines, 56(3), 345-365. https://doi.org/10.1111/jcpp.12381

Powell, B. J., McMillen, J. C., Hawley, K. M., \& Proctor, E. K. (2013). Mental health clinicians' motivation to invest in training: Results from a practice-based research network survey. Psychiatric Services, 64(8), 816-818. https://doi.org/10.1176/appi.ps.003602012

Powell, B. J., Mcmillen, J. C., Proctor, E. K., Christopher, R., Griffey, R. T., Bunger, A. C., ... York, J. L. (2013). A compilation of strategies for implementing clinical innovations in health and mental health. Medical Care Research and Revieware Research and Review, 69(2), 1-28. https://doi.org/10.1177/1077558711430690.A

Powell, B. J., Proctor, E. K., \& Glass, J. E. (2013). A systematic review of strategies for implementing empirically supported mental health interventions. Research on Social Work Practice. https://doi.org/10.1177/1049731513505778

Powell, B. J., Waltz, T. J., Chinman, M. J., Damschroder, L. J., Smith, J. L., Matthieu, M. M., ... Kirchner, J. E. (2015). A refined compilation of implementation strategies: results from the Expert Recommendations for Implementing Change (ERIC) project. Implementation Science, 10(1), 21. https://doi.org/10.1186/s13012-015-0209-1

Proctor, E. K., Landsverk, J., Aarons, G., Chambers, D., Glisson, C., \& Mittman, B. (2009). 
Implementation research in mental health services: an emerging science with conceptual, methodological, and training challenges. Administration and Policy in Mental Health and Mental Health Services Research, 36(1), 24-34.

Proctor, E., Silmere, H., Raghavan, R., Hovmand, P., Aarons, G., Bunger, A., ... Hensley, M. (2011). Outcomes for implementation research: Conceptual distinctions, measurement challenges, and research agenda. Administration and Policy in Mental Health and Mental Health Services Research, 38(2), 65-76. https://doi.org/10.1007/s10488-010-0319-7

Rodríguez, A., Southam-Gerow, M. A., O’Connor, M. K., \& Allin, R. B. (2014). An analysis of stakeholder views on children's mental health services. Journal of Clinical Child \& Adolescent Psychology, 43(6), 862-876. https://doi.org/10.1080/15374416.2013.873982

Saldana, L., Chapman, J. E., Henggeler, S. W., \& Rowland, M. D. (2007). The Organizational Readiness for Change scale in adolescent programs: Criterion validity. Journal of Substance Abuse Treatment, 33(2), 159-169. https://doi.org/10.1016/j.jsat.2006.12.029

Schell, S. F., Luke, D. A., Schooley, M. W., Elliott, M. B., Herbers, S. H., Mueller, N. B., \& Bunger, A. C. (2013). Public health program capacity for sustainability: A new framework. Implementation Science, 8(1), 1-9. https://doi.org/10.1186/1748-5908-8-15

Scheirer, M. A. (2005). Is sustainability possible? A review and commentary on empirical studies of program sustainability. American Journal of Evaluation, 26(3), 320-347. https://doi.org/10.1177/1098214005278752

Scheirer, M. A., \& Dearing, J. W. (2011). An agenda for research on the sustainability of public health programs. American Journal of Public Health, 101(11), 2059-2067. https://doi.org/10.2105/AJPH.2011.300193

Schneider, B., Ehrhart, M. G., \& Macey, W. H. (2013). Organizational climate and 
culture. Annual Review of Psychology, 64, 361-388.

Schoenwald, S. K., Sheidow, A. J., \& Letourneau, E. J. (2004). Toward effective quality assurance in evidence-based practice: Links between expert consultation, therapist fidelity, and child outcomes. Journal of Clinical Child \& Adolescent Psychology, 33(1), 94-104. https://doi.org/10.1207/S15374424JCCP3301_10

Shore, B. A., Iwata, B. A., Vollmer, T. R., Lerman, D. C., \& Zarcone, J. R. (1995). Pyramidal staff training in the extension of treatment for severe behavior disorders. Journal of Applied Behavior Analysis, 28(3), 323-332. https://doi.org/10.1901/jaba.1995.28-323

Silverman, W. K., Pina, A. A., \& Viswesvaran, C. (2008). Evidence-based psychosocial treatments for phobic and anxiety disorders in children and adolescents. Journal of Clinical Child and Adolescent Psychology, 37(1), 105-130. https://doi.org/10.1080/15374410701817907

Solberg, L. I., Kottke, T. E., Brekke, M. L., Magnan, S., Davidson, G., Calomeni, C. A., ... Nelson, A. F. (2000). Failure of a continuous quality improvement intervention to increase the delivery of preventive services. A randomized trial. Effective Clinical Practice, 3(3), $105-115$.

Southam-Gerow, M. A., Chorpita, B. F., Miller, L. M., \& Gleacher, A. A. (2008). Are children with anxiety disorders privately referred to a university clinic like those referred from the public mental health system? Administration and Policy in Mental Health and Mental Health Services Research, 35(3), 168-180. https://doi.org/10.1007/s10488-007-0154-7

Southam-Gerow, M. A., Hourigan, S. E., \& Allin, R. B. (2009). Adapting evidence-based mental health treatments apartnership approach. Behavior Modification, 33(1), 82-103. https://doi.org/10.1177/0145445508322624 
Southam-Gerow, M. A., Weisz, J. R., \& Kendall, P. C. (2003). Youth with anxiety disorders in research and service clinics: Examining client differences and similarities. Journal of Clinical Child and Adolescent Psychology, 32(3), 375-385. https://doi.org/10.1207/S15374424JCCP3203_06

Steele, R. G., Elkin, D. T., \& Roberts, M. C. (2008). Handbook of evidence-based therapies for children and adolescents: Bridging science and practice. New York, NY: Springer Science \& Business Media.

Stewart, R. E., \& Chambless, D. (2007). Does psychotherapy research inform treatment decisions in private practice. Journal of Clinical Psychology, 66(4), 430-441.

Stirman, S., Kimberly, J., Cook, N., Calloway, A., Castro, F., \& Charns, M. (2012). The sustainability of new programs and innovations: a review of the empirical literature and recommendations for future research. Implementation Science, 7(1), 17.

\section{https://doi.org/10.1186/1748-5908-7-17}

Swain, K., Whitley, R., McHugo, G. J., \& Drake, R. E. (2010). The sustainability of evidencebased practices in routine mental health agencies. Community Mental Health Journal, 46(2), 119-129. https://doi.org/10.1007/s10597-009-9202-y

Tibbits, M. K., Bumbarger, B. K., Kyler, S. J., \& Perkins, D. F. (2010). Sustaining evidencebased interventions under real-world conditions: Results from a large-scale diffusion project. Prevention Science, 11(3), 252-262. https://doi.org/10.1007/s11121-010-0170-9

University of California, Davis. (2014). PCIT Web Course. Retrieved from https://pcit.ucdavis.edu/pcit-web-course/

U.S. Department of Health and Human Services. (2003). Achieving the promise: Transforming mental health care in America (DHHS Publication No. SMA 03-3822). Rockville, MD: 


\section{U.S. Government Printing Office.}

U.S. Public Health Service. (2000). Report of the surgeon general's conference on children's mental health: A national action agenda. Washington, District of Columbia. Retrieved from http://www.ncbi.nlm.nih.gov/books/NBK44233/

Waldron, H. B., \& Turner, C. W. (2008). Evidence-based psychosocial treatments for adolescent substance abuse. Journal of Clinical Child and Adolescent Psychology, 37(1), 238-261. https://doi.org/10.1080/15374410701820133

Wampold, B. E., Budge, S. L., Laska, K. M., del Re, A. C., Baardseth, T. P., Fluckiger, C., ... Gunn, W. (2011). Evidence-based treatments for depression and anxiety versus treatmentas-usual: A meta-analysis of direct comparisons. Clinical Psychology Review, 31(8), 13041312. https://doi.org/10.1016/j.cpr.2011.07.012

Wang, W., Saldana, L., Brown, C. H., \& Chamberlain, P. (2010). Factors that influenced county system leaders to implement an evidence-based program: A baseline survey within a randomized controlled trial. Implementation Science, 5(1), 72.

Weersing, V. R., Jeffreys, M., Do, M. C. T., Schwartz, K. T., \& Bolano, C. (2017). Evidence base update of psychosocial treatments for child and adolescent depression. Journal of Clinical Child \& Adolescent Psychology, 46(1), 11-43.

Williams, N. J., Glisson, C., Hemmelgarn, A., \& Green, P. (2017). Mechanisms of change in the ARC organizational strategy: increasing mental health clinicians' EBP adoption through improved organizational culture and capacity. Administration and Policy in Mental Health and Mental Health Services Research, 44(2), 269-283.

Williams, N. J., Ehrhart, M. G., Aarons, G. A., Marcus, S. C., \& Beidas, R. S. (2018). Linking molar organizational climate and strategic implementation climate to clinicians' use of 
evidence-based psychotherapy techniques: cross-sectional and lagged analyses from a 2year observational study. Implementation Science, 13(1), 85. 
Table 1

Baseline Descriptive Statistics of Participants

\begin{tabular}{l|ccc|ccc}
\hline \multirow{2}{*}{} & \multicolumn{3}{|c|}{ Clinicians } & \multicolumn{3}{c}{ Administrators } \\
\cline { 2 - 7 } & $N$ & $N$ Yes & Percent & $N$ & $N$ Yes & Percent \\
\hline Gender (\% female) & 100 & 84 & $84.0 \%$ & 50 & 31 & $62.0 \%$ \\
Professionally licensed (\% yes) & 100 & 52 & $52.0 \%$ & $N / A$ & $N / A$ & $N / A$ \\
Minority status (\% minority) & 99 & 14 & $14.1 \%$ & 49 & 6 & $12.2 \%$ \\
Part or full time (\% full time) & 100 & 72 & $72.0 \%$ & $N / A$ & $N / A$ & $N / A$ \\
Years worked at agency (\% $>3$ yrs) & 100 & 46 & $46.0 \%$ & 49 & 41 & $83.7 \%$ \\
\hline Type (highest) degree earned & & & & & & \\
$\quad$ Education & 100 & 2 & $2.0 \%$ & 48 & 3 & $6.3 \%$ \\
$\quad$ Medicine & 100 & 4 & $4.0 \%$ & 48 & 3 & $6.3 \%$ \\
$\quad$ Nursing & 100 & 0 & $0.0 \%$ & 48 & 0 & $0.0 \%$ \\
$\quad$ Psychology & 100 & 37 & $37.0 \%$ & 48 & 11 & $22.9 \%$ \\
$\quad$ Social Work & 100 & 31 & $31.0 \%$ & 48 & 16 & $33.3 \%$ \\
$\quad$ Other & 100 & 26 & $26.0 \%$ & 48 & 15 & $31.3 \%$ \\
\hline & $N$ & Mean & $S D$ & $N$ & Mean & $S D$ \\
\hline Age at baseline & 100 & 39.0 & 10.0 & 49 & 48.5 & 9.0 \\
Years experience in field & 100 & 11.4 & 8.2 & 49 & 22.2 & 8.5 \\
\hline
\end{tabular}




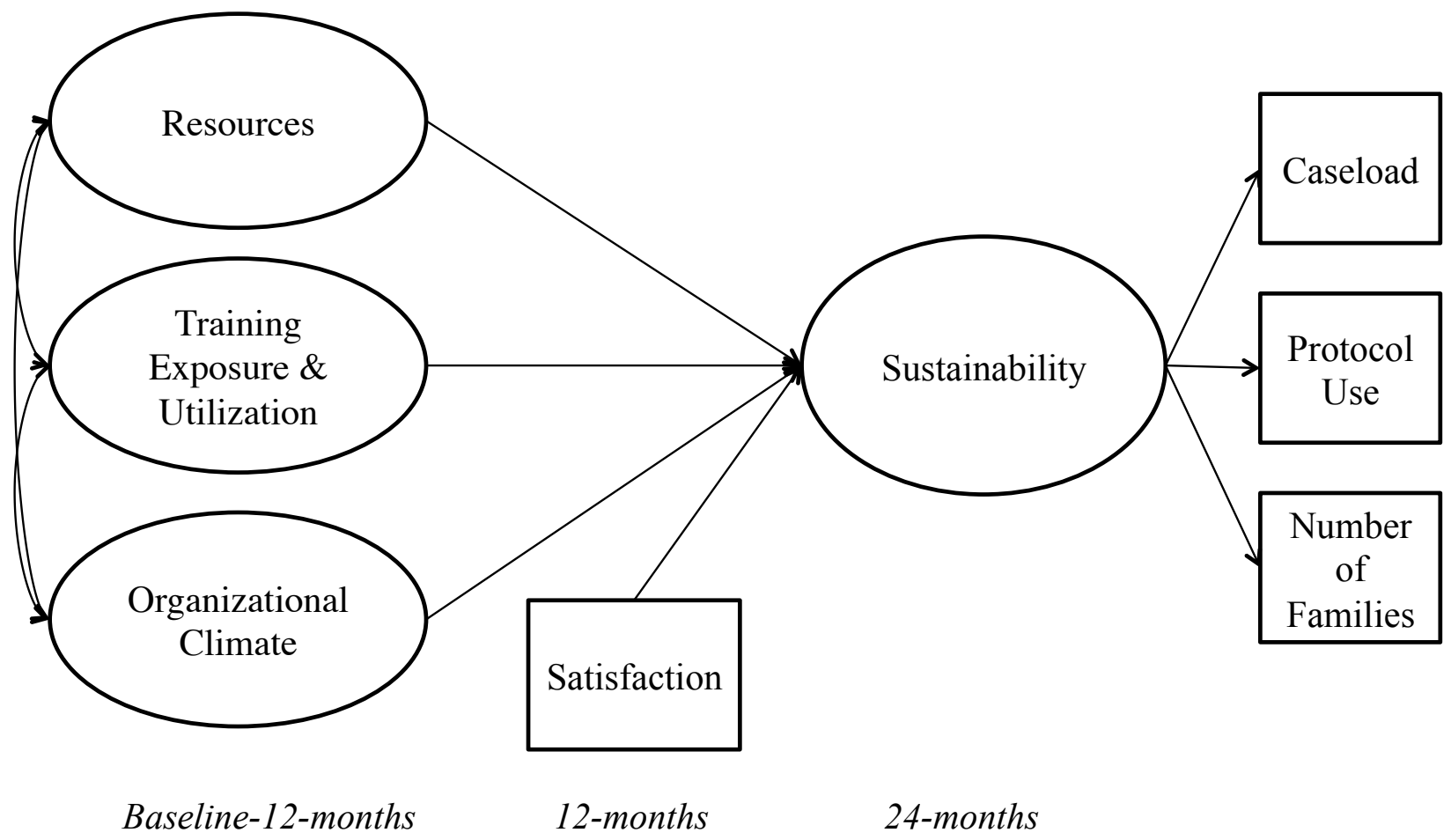

Figure 1. Proposed model for aim two, the effect of organizational barriers on sustainability. 


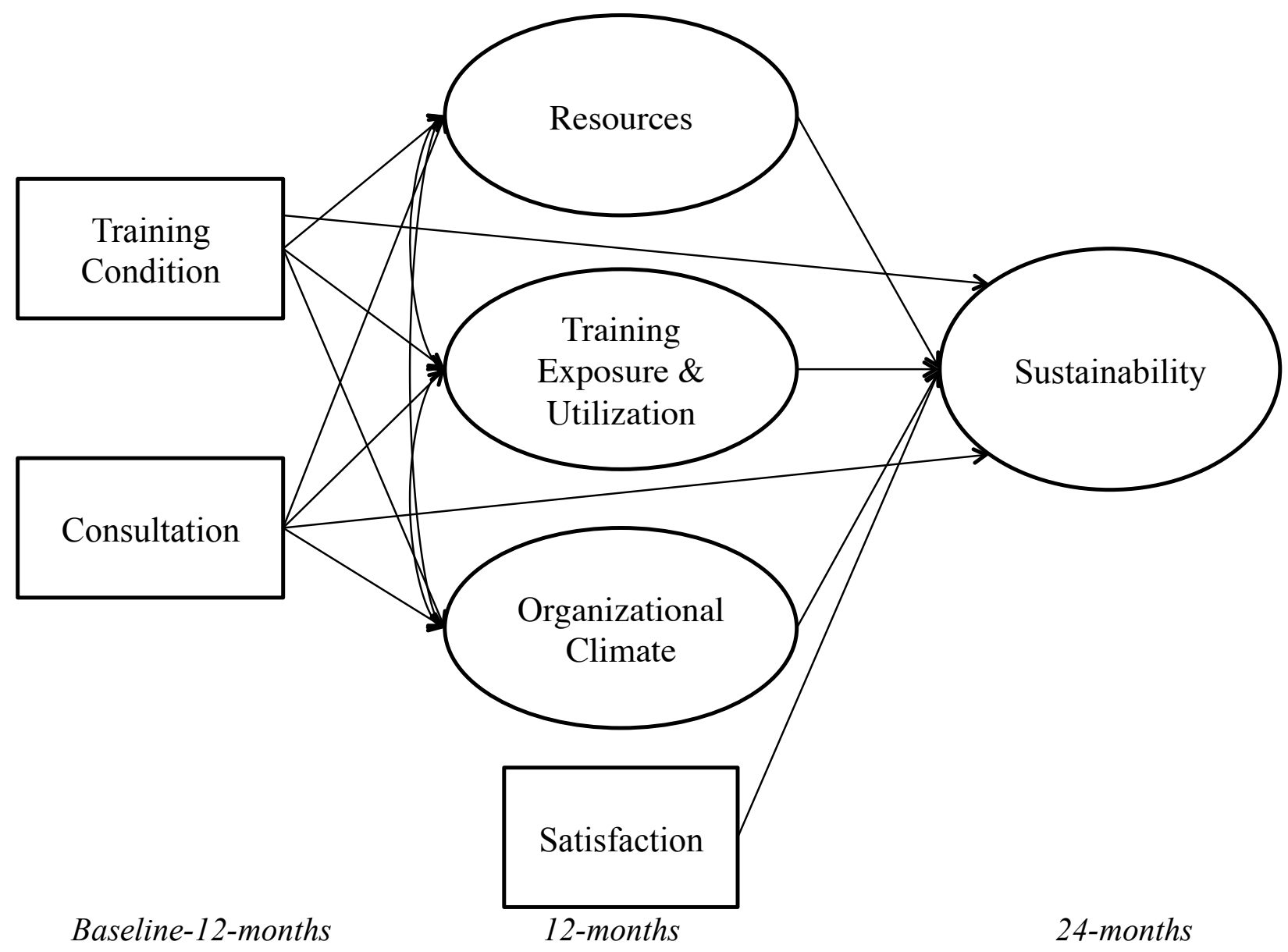

Figure 2. Proposed model for aim three, indirect effect of training design and consultation calls on sustainability. 
Table 2

Correlations Between Administrator and Clinician Report of Sustainability

\begin{tabular}{lccccc}
\hline Variables & 1 & 2 & 3 & 4 & 5 \\
\hline 1. Administrator: Still offering PCIT & & & & & \\
2. Administrator: Serving __ clients & $.25^{*}$ & & & & \\
3. Administrator: Approximate caseload & .19 & $.26^{*}$ & & & \\
4. Clinician: Approximate caseload & -.06 & -.13 & $.44^{*}$ & & \\
5. Clinician: Number of families & .10 & -.04 & $.38^{*}$ & $.56^{*}$ & \\
6. Clinician: Use of protocol & $-.49^{* *}$ & -.13 & -.17 & -.15 & $-.28^{*}$ \\
\hline
\end{tabular}

Note: $* p<.05, * * p<.01$ 


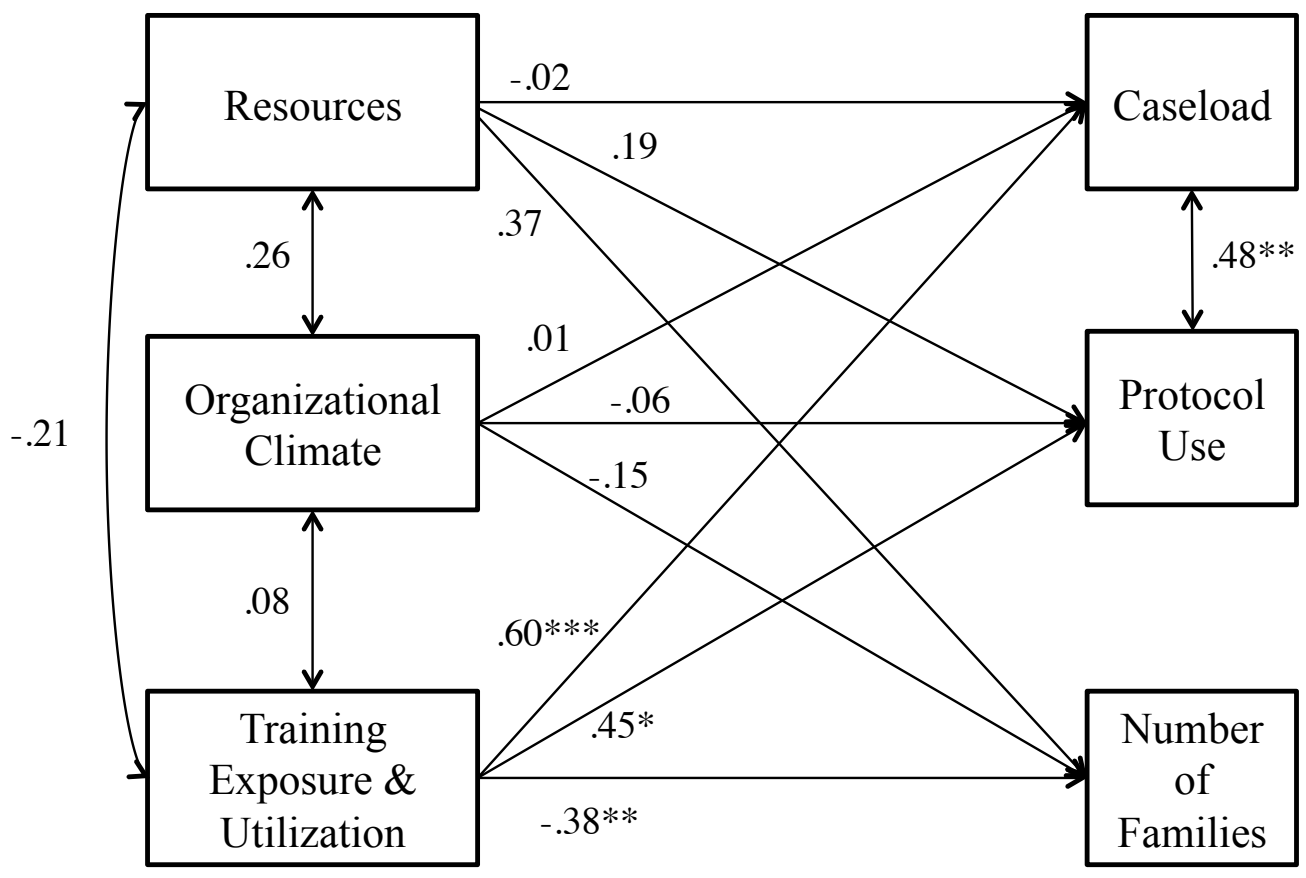

Baseline

24-months

Figure 3. Model for aim two, baseline organizational characteristics on sustainability, including standardized estimates. (Note: $* p<.05, * * p<.01, * * * p<.001$ ) 


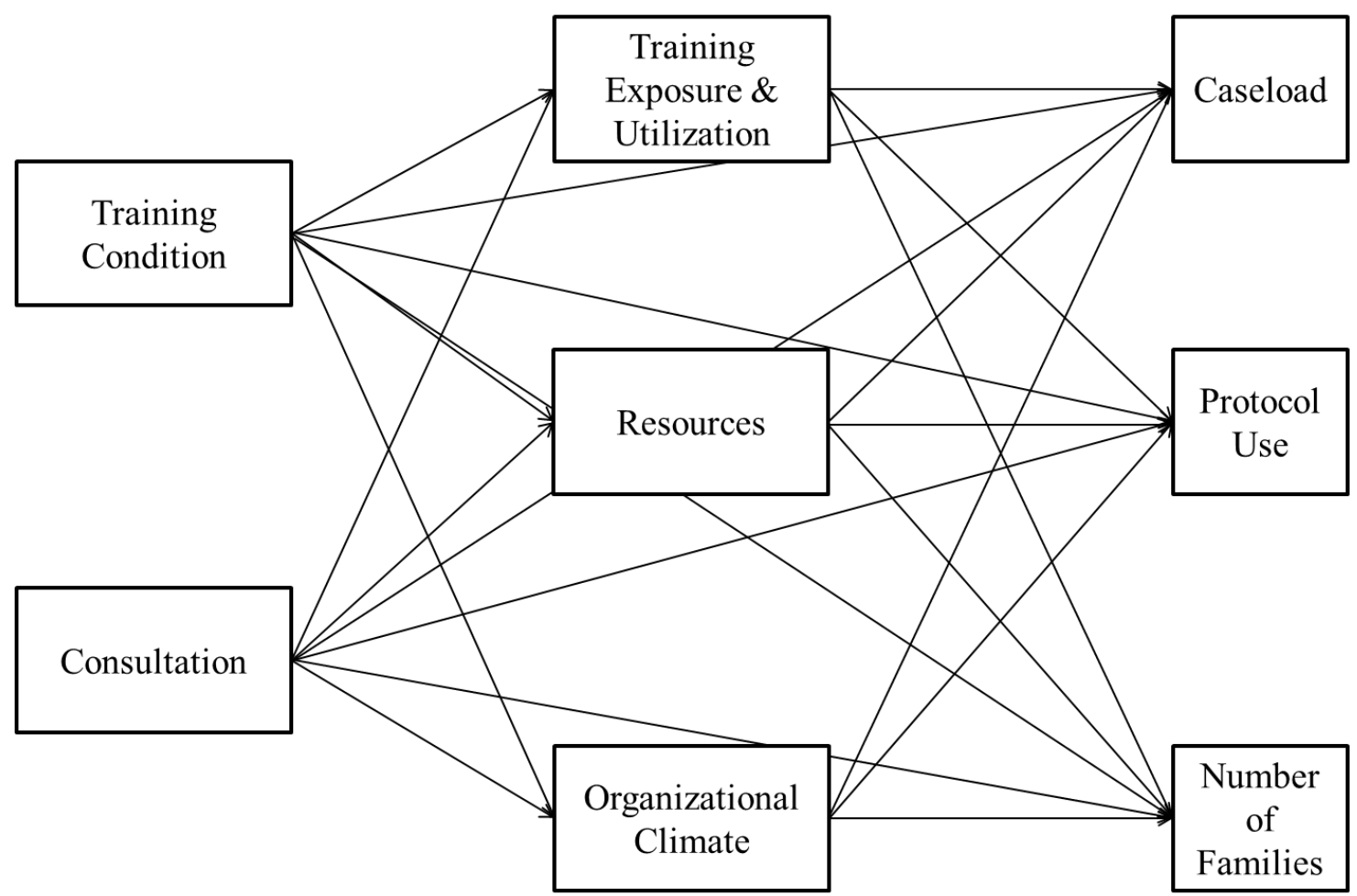

Figure 4. Model for aim three, the direct and indirect effect of training and consultation on sustainability. 


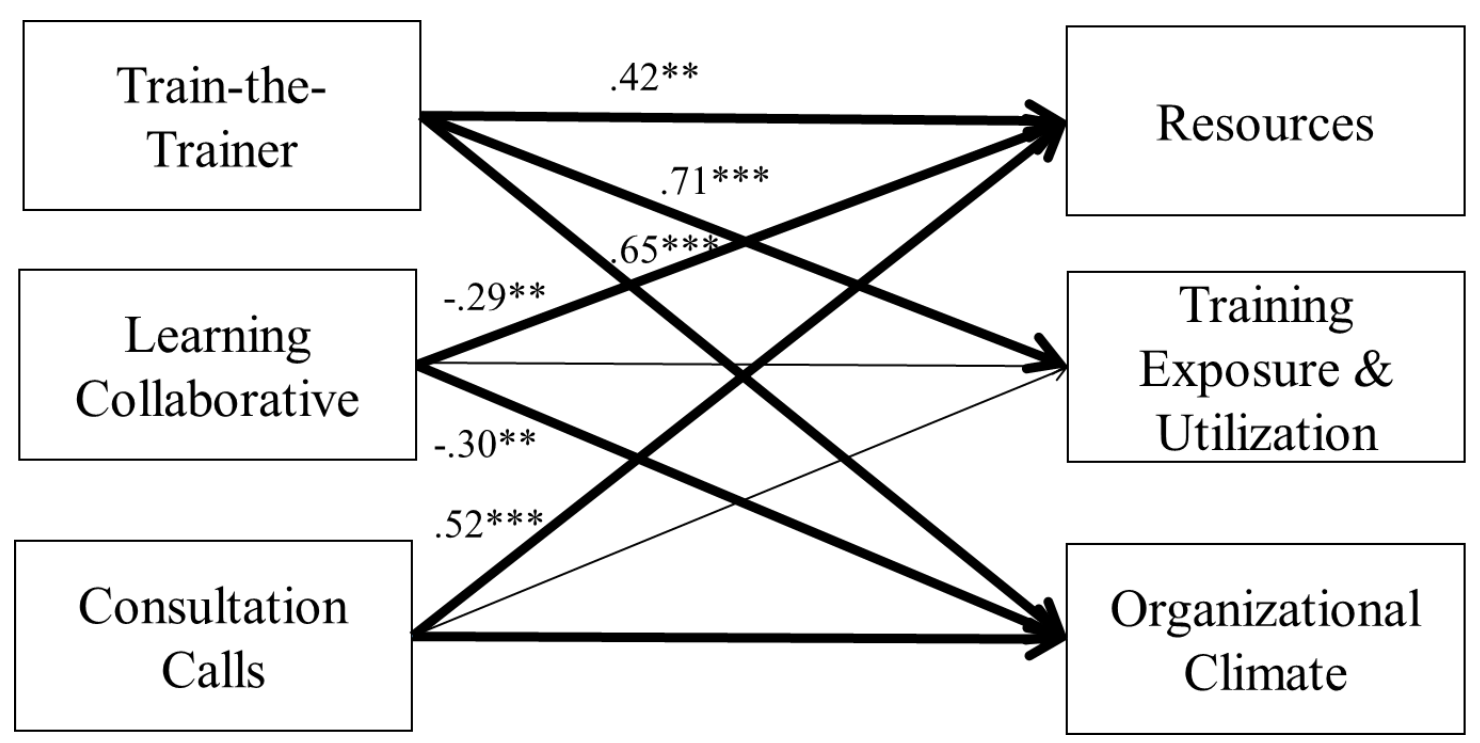

Figure 5. Associations among training condition, consultation calls, and organizational variables (Note: Significant paths are in bold). 


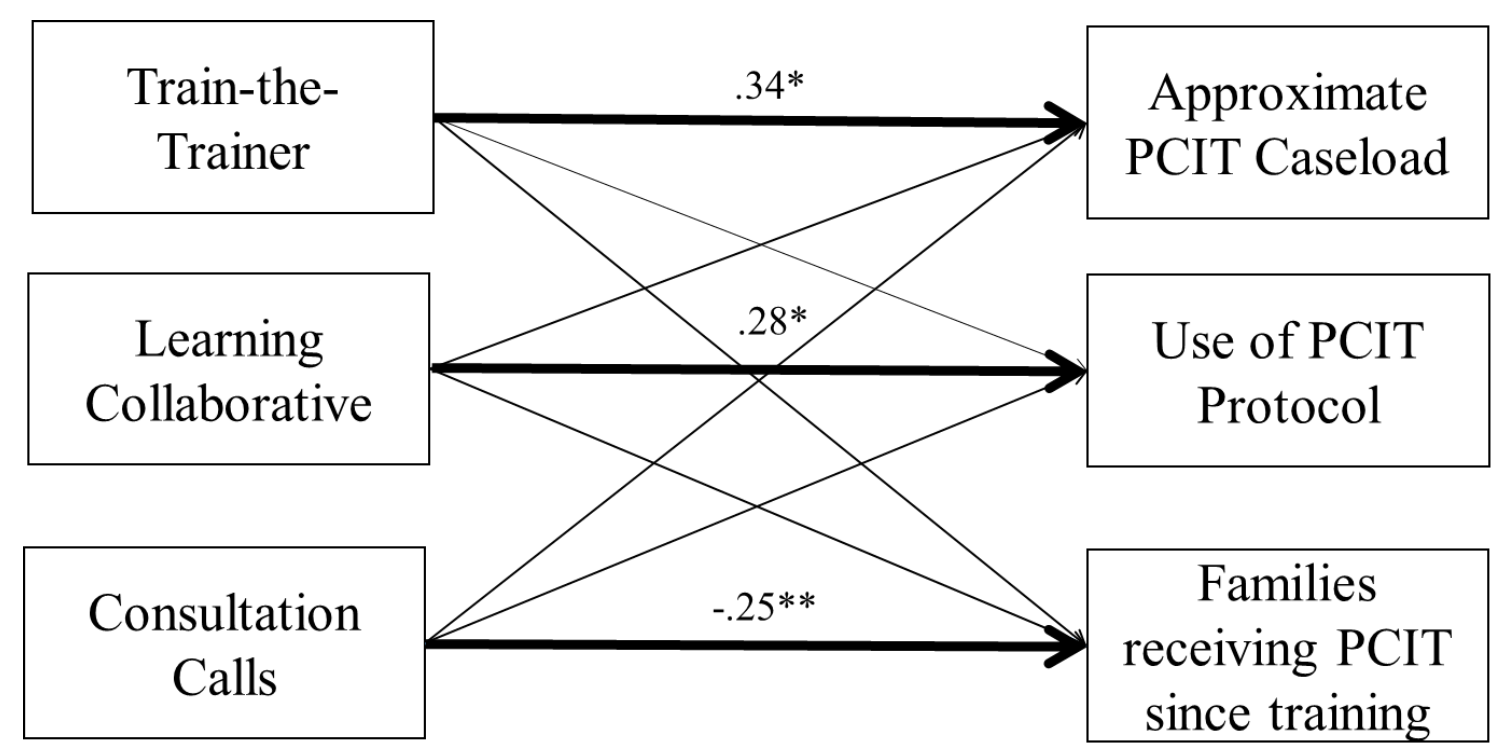

Figure 6. Associations among training condition, consultation calls, and organizational variables (Note: Significant paths are in bold). 